Opinion paper

\title{
The search for noise-induced cochlear synaptopathy in humans: Mission impossible?
}

\author{
Naomi Bramhall a, b, Elizabeth Francis Beach ${ }^{\mathrm{c}, \mathrm{d}}$, Bastian Epp ${ }^{\mathrm{e}}$, Colleen G. Le Prell ${ }^{\mathrm{f}}$, \\ Enrique A. Lopez-Poveda ${ }^{g}$, Christopher J. Plack ${ }^{\text {h, i }}$, Roland Schaette ${ }^{\mathrm{j}}$, Sarah Verhulst ${ }^{\mathrm{k}, \mathrm{l}}$, \\ Barbara Canlon ${ }^{\mathrm{m}}$,
}

a VA RR\&D National Center for Rehabilitative Auditory Research (NCRAR), VA Portland Health Care System, Portland, OR, USA

${ }^{\mathrm{b}}$ Department of Otolaryngology/Head and Neck Surgery, Oregon Health \& Science University, Portland, OR, USA

c National Acoustic Laboratories, Macquarie University, Australia

d The HEARing CRC, Melbourne, Australia

e Hearing Systems Group, Department of Electrical Engineering, Technical University of Denmark, Denmark

${ }^{\mathrm{f}}$ School of Behavioral and Brain Sciences, The University of Texas at Dallas, USA

${ }^{\mathrm{g}}$ Instituto de Neurociencias de Castilla y Leon, Departamento de Cirugía, University of Salamanca, 37007, Salamanca, Spain

${ }^{\mathrm{h}}$ Manchester Centre for Audiology and Deafness, The University of Manchester, Manchester Academic Health Science Centre, M13 9PL, UK

${ }^{i}$ Department of Psychology, Lancaster University, LA1 4YF, UK

j UCL Ear Institute, 332 Gray's Inn Road, London, WC1X 8EE, UK

${ }^{\mathrm{k}}$ Hearing Technology @ Waves, Dept. of Information Technology, Ghent University, Belgium

${ }^{1}$ Technologiepark 15, 9052, Zwijnaarde, BE, Belgium

${ }^{\mathrm{m}}$ Department of Physiology and Pharmacology, Karolinska Institute, Stockholm, 171 77, Sweden

\section{A R T I C L E I N F O}

\section{Article history:}

Received 21 November 2018

Received in revised form

25 February 2019

Accepted 28 February 2019

Available online 9 March 2019

\begin{abstract}
A B S T R A C T
Animal studies demonstrate that noise exposure can permanently damage the synapses between inner hair cells and auditory nerve fibers, even when outer hair cells are intact and there is no clinically relevant permanent threshold shift. Synaptopathy disrupts the afferent connection between the cochlea and the central auditory system and is predicted to impair speech understanding in noisy environments and potentially result in tinnitus and/or hyperacusis. While cochlear synaptopathy has been demonstrated in numerous experimental animal models, synaptopathy can only be confirmed through postmortem temporal bone analysis, making it difficult to study in living humans. A variety of noninvasive measures have been used to determine whether noise-induced synaptopathy occurs in humans, but the results are conflicting. The overall objective of this article is to synthesize the existing data on the functional impact of noise-induced synaptopathy in the human auditory system. The first section of the article summarizes the studies that provide evidence for and against noise-induced synaptopathy in humans. The second section offers potential explanations for the differing results between studies. The final section outlines suggested methodologies for diagnosing synaptopathy in humans with the aim of improving consistency across studies.
\end{abstract}

(C) 2019 Elsevier B.V. All rights reserved.

\section{Introduction}

Damage to the inner ear, and associated hearing loss, occurs

\footnotetext{
* Corresponding author.

E-mail addresses: Naomi.Bramhall@va.gov (N. Bramhall), elizabeth.beach@nal. gov.au (E.F. Beach), bepp@elektro.dtu.dk (B. Epp), Colleen.LePrell@utdallas.edu (C.G. Le Prell), ealopezpoveda@usal.es (E.A. Lopez-Poveda), chris.plack@ manchester.ac.uk (C.J. Plack), r.schaette@ucl.ac.uk (R. Schaette), s.verhulst@ugent. be (S. Verhulst), Barbara.Canlon@ki.se (B. Canlon).
}

from noise trauma, ototoxic drugs, aging and genetic factors. One form of cochlear pathology involves synaptic damage that perturbs the neurotransmission between the inner hair cell (IHC) and auditory nerve (AN) fibers. This type of pathology has been termed cochlear synaptopathy (Kujawa and Liberman, 2015) and popularly "hidden hearing loss" (Schaette and McAlpine, 2011) because it can occur without affecting hearing thresholds. However, the definition of the latter term has become inconsistent between articles, with some authors using it to refer more generally to hearing dysfunction in the presence of normal hearing thresholds. For this reason, 
we will avoid use of the term "hidden hearing loss" in the present review. The predicted functional consequences of these synaptic alterations are listening difficulties in noisy backgrounds, tinnitus and hyperacusis (Kujawa and Liberman, 2015). Experimental work, primarily on noise-traumatized and aging rodents, has clearly demonstrated that the afferent synapse is more vulnerable than hair cells. AN fibers with low and medium spontaneous rates (SRs) and higher response thresholds appear to be particularly vulnerable to noise damage (Furman et al., 2013). Since these fibers do not respond at low intensity levels, their loss does not impact measures of auditory threshold, such as the clinical audiogram. Although auditory brainstem response (ABR) thresholds are insensitive to these synaptic changes, and there is some evidence that low-SR fibers do not contribute to the amplitude of ABR wave 1 (Bourien et al., 2014), the amplitude of ABR wave 1 recorded to suprathreshold transients appears to be a sensitive indicator of synaptopathy (Kujawa and Liberman, 2015; Furman et al., 2013). The amplitude of the middle-ear muscle reflex (MEMR) and the envelope following response (EFR) also appear to be sensitive to synaptopathy in animal models (Shaheen et al., 2015; Valero et al. 2016, 2018). Loss of low-SR fibers may degrade the representation of high-intensity sounds, although an alternative account is that the low-SR fibers are more involved in efferent regulation than in highintensity coding (Carney, 2018).

It has recently been questioned whether cochlear synaptopathy occurs in humans and if there is evidence for functional consequences of this phenomenon, as revealed by listening difficulties in noisy backgrounds, tinnitus or hyperacusis. The purpose of this article is to highlight the research that finds evidence supporting noise-induced human synaptopathy, contrast this with studies that have not provided supporting evidence, discuss possible reasons for null results and diverging outcomes, and provide guidance to the field regarding research protocols. To outline these inconsistencies, the existing data that either support or do not support that noise-induced synaptopathy occurs in humans are summarized. Details of the cited studies can be found in Table 1. Next, possible explanations for these inconsistencies are provided. Finally, the last part of the article discusses methodological considerations for diagnosing synaptopathy in humans in order to standardize future experimental approaches. This will facilitate the integration of data across studies and improve the overall understanding of cochlear synaptopathy in humans.

\subsection{Data consistent with noise-induced synaptopathy in humans}

\subsubsection{Post-mortem temporal bone studies}

There is little debate regarding the existence of age-related synaptic loss (or synaptopathy) in the human inner ear. Analysis of temporal bones harvested post-mortem demonstrate that both age-related synaptopathy (Viana et al., 2015) (Fig. 1) and agerelated neural degeneration can occur in humans (Makary et al., 2011). In the study by Makary et al., temporal bones were carefully selected to include only those with no overt loss of either IHCs or outer hair cells (OHCs), demonstrating that spiral ganglion cell numbers can decrease prior to hair cell loss. The results of Viana et al. suggest that synaptopathy can also occur independently of hair cell loss. The Viana et al. study included temporal bones with a combination of synaptic loss and hair cell (IHC and OHC) loss, with the most extreme hair cell loss observed in the cochlear base. Interestingly, a temporal bone from a female (age 67) donor had a notched OHC loss centered at $3000 \mathrm{~Hz}$, which is suggestive of previous noise injury. Fewer type I fibers/IHC and fewer synapses/ IHC were observed in this donor relative to a 54-year-old male and a 70-year-old female donor, which is consistent with noise-related neuronal/synaptic loss. These findings build on earlier observations of age-related AN fiber loss in temporal bones that also had "expected” age-related loss of OHCs (Felder and Schrott-Fischer, 1995). Wu et al. (2018) also demonstrated significant age-related synapse and AN fiber loss in ears with expected age-related loss of hair cells. In addition, although spiral ganglion cell loss can occur independently of hair cell loss, neuronal loss is greater when hair cells are also missing (Otte et al., 1978). Thus, cochlear synaptopathy and neuropathy may be some of the earliest manifestations of future sensorineural hearing loss (SNHL) where synaptic/neuronal loss coexists with hair cell loss.

\subsubsection{Human auditory brainstem response studies of noise-induced synaptopathy}

To date, the main electrophysiological metric for studying synaptopathy in humans has been the amplitude of wave I of the ABR, a measure of AN function that is associated with synaptopathy in rodent models (Kujawa and Liberman, 2009; Sergeyenko et al., 2013). In a sample of young (age 19-35) military veterans and non-veterans with normal audiograms and good distortionproduct otoacoustic emissions (DPOAEs), Bramhall et al. (2017) found a reduction in ABR wave I amplitude for veterans with high levels of reported noise exposure during their military service and non-veterans who reported firearm use compared to non-veterans with less noise exposure (Fig. 2). Liberman et al. (2016) showed an increase in the amplitude ratio of the ABR summating potential (SP) to the action potential (AP; equivalent to wave I of the $A B R$ ) in college music students (age 18-41) with high levels of reported noise exposure versus non-music students with lower reported noise exposure histories (Fig. 3). It must be noted that using the SP/ AP ratio can be problematic as a normalizing strategy since the ratio is critically dependent on changes in the numerator. Stamper and Johnson (2015a) reported a reduction in ABR wave I amplitude for young people (age 19-28) with higher reported recreational noise exposure compared to individuals with lower exposure, but a reanalysis showed that this relationship held true only for female participants (Stamper and Johnson, 2015b). In a group of older adults (aged 29-55) with pure tone thresholds ranging from normal to mild hearing loss, Valderrama et al. (2018) reported a significant relationship between lower ABR wave I amplitude and increasing lifetime noise exposure.

\subsubsection{Human envelope following response studies of noise-induced synaptopathy}

The EFR is an evoked potential generated in response to amplitude modulated sounds (often a sinusoidally amplitude modulated pure tone) that can be measured from electrodes placed on the scalp. The EFR provides an indication of the fidelity with which the auditory system can follow the envelope of a stimulus (Dolphin and Mountain, 1992). Two studies demonstrated that EFR strength was reduced in mice with histologically confirmed synapatopathy that was either induced through aging (Parthasarathy and Kujawa, 2018) or noise exposure (Shaheen et al., 2015). The EFR was most sensitive to synaptopathy for stimulus modulation frequencies between 700 and $1000 \mathrm{~Hz}$, which is consistent with the EFR being generated at the AN. However, it is unclear how these animal results will translate to humans, where the EFR is generally measured at much lower modulation frequencies $(80-120 \mathrm{~Hz})$, targeting EFR generators from the auditory midbrain. Bharadwaj et al. (2015) detected a marginally significant difference in the EFR slope and envelope interaural time difference (ITD) threshold when participants were grouped as "more" and "less" noise exposed, but argued this result should be interpreted with caution based on the crude characterization of noise-exposure history, small sample size, and cross-correlations among the temporal coding outcomes. While simulation studies based on functional 
Main Results

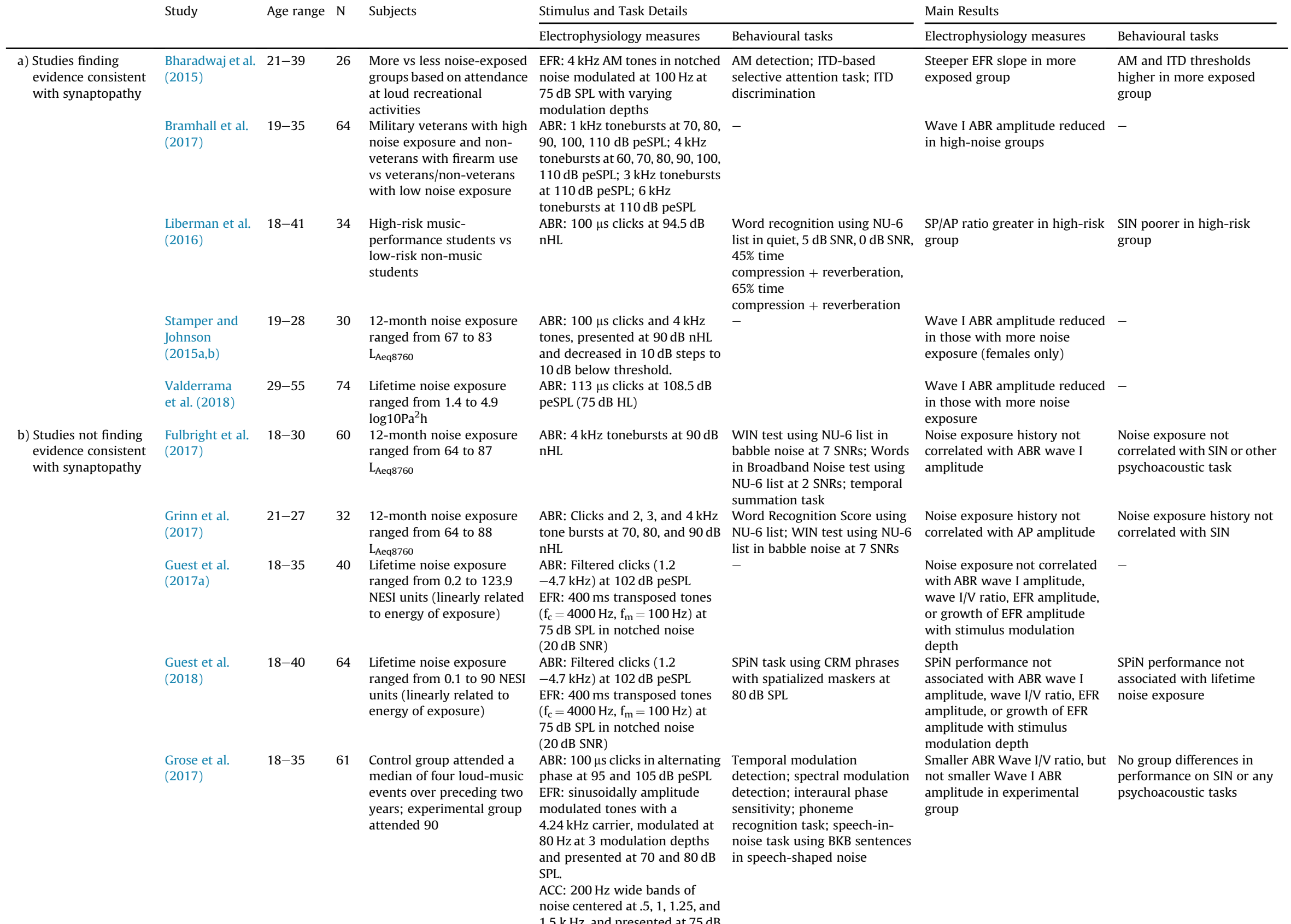


SPL, with and without

interruption

ABR: $100 \mu$ s filtered clicks at 80

and $100 \mathrm{~dB}$ peSPL

FFR: A low-frequency tone and

a transposed tone presented to

each ear presented at $80 \mathrm{~dB}$ SPL

\section{Prendergast \\ $18-36$ \\ ranged from 0 to 2.5} $\log 10$ (Energy)

$\log 10$ (Energy)

Prendergast
et al. (2018)

Ridley et al.

Spankovich

et al. (2017)

Yeend et al.

(2017)
19-34

30 Low vs high lifetime exposure groups: 3-0.5 og10 (energy) for low nois

group; high noise group

33 12-month noise exposure ranged from 66.8 to 86.5 $\mathrm{L}_{\text {Aeq8760 }}$

$18-28$

2anth noise exposu

$\mathrm{L}_{\mathrm{Aeq} 8760}$

30-57 122 Lifetime noise exposure

ranged from 1.4 to 4.9 $\log 10 \mathrm{~Pa}^{2} \mathrm{~h}$
$1.1-2.6 \log 10$ (energy) for

(115.5 dB peSPL)
Frequency difference limens;

intensity difference limens;

interaural phase difference

discrimination; amplitude

discrimination, amplitude

triplet test; SPIN task using

CRM phrases with maskers:

localisation task

ABR: $100 \mu$ s clicks at $80 \mathrm{~dB} \mathrm{nHL}$

ABR: $1 \mathrm{msec}$ tonebursts at 1 and
$4 \mathrm{kHz}$ presented at 80 and

$100 \mathrm{~dB}$ peSPL

Thresholds in noise tested with
the TEN (HL) test; Categorical loudness scaling

ABR: $100 \mu$ s clicks at $75 \mathrm{~dB} \mathrm{nHL}$

$(106.7 \mathrm{~dB}$ peSPL) at 8

presentation rates

50,65 , and $80 \mathrm{~dB}$ nHL at 2

presentation rates

Tones in noise detection;

temporal fine structure tes

amplitude modulation

detection; LiSN-S test; dynamic

conversations test
Noise exposure not correlated -

with ABR Wave I amplitude

correlated with

performance on any

psychoacoustic tasks

Lifetime noise exposure

unrelated to SP/AP or ABR wave

I amplitude

Abbreviations: $\mathrm{ACC}=$ acoustic change complex; $\mathrm{AM}=$ amplitude modulation; $\mathrm{ABR}=$ auditory brainstem response; $\mathrm{BKB}=$ Bamford-Kowel-Bench; $\mathrm{dB}$ peSPL= peak-to-peak equivalent $\mathrm{SPL}$ (or peak equivalent $\mathrm{SPL}$ );

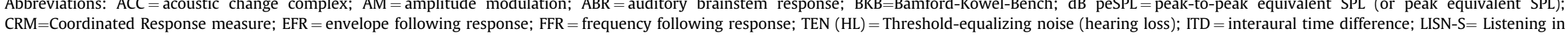
$\mathrm{CRM}=$ Coordinated Response measure; EFR = envelope following response; $\mathrm{FFR}=$ frequency following response; TEN $(\mathrm{HL})=\mathrm{Threshold}$-equalizing noise (hearing loss); ITD = interaura
Spatialized Noise-Sentences; NESI= Noise Exposure Structured Interview; SIN=Speech in noise; SNR = signal to noise ratio; SPiN = Speech perception in noise; WIN = words in noise. 

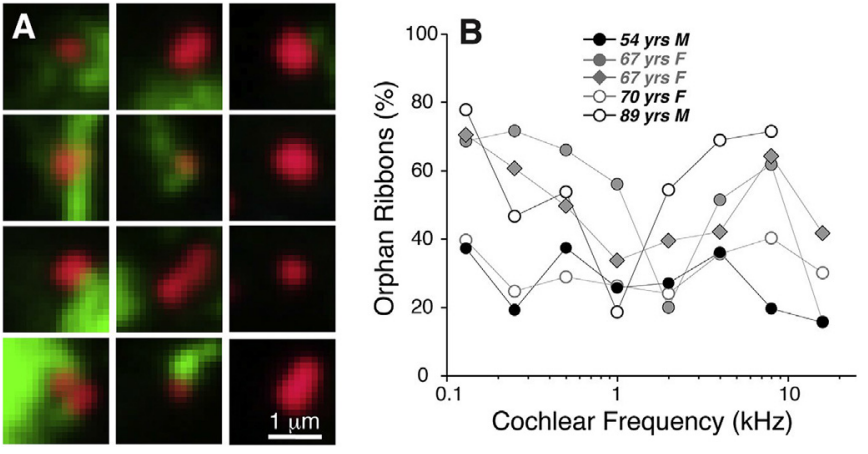

Fig. 1. Histological evidence of synaptopathy in human temporal bones.

Figure shows analysis of orphan ribbons in the IHC area. A: Thumbnail re-projections of the voxel space immediately surrounding 12 selected synaptic ribbons from zstacks. Some ribbons are clearly juxtaposed to nerve terminals (left two columns) while others are not (right column). Only the red (anti-CtBP2) and green (anti-neurofilament) channels are shown for clarity. B: Percentage of orphan ribbons, i.e. those not closely juxtaposed to post-synaptic terminals, as assessed by evaluating thumbnail arrays such as those illustrated in $\mathbf{A}$, for each of the five completely reconstructed ears in the present study. Reprinted with permission from Viana et al., 2015, Hearing Research. (For interpretation of the references to colour in this figure legend, the reader is referred to the Web version of this article.)

models of the auditory periphery show a role for synaptopathy in reduced EFR strength (Bharadwaj et al., 2014; Paul et al., 2017; Verhulst et al. 2018a, 2018b), it remains unclear whether the EFR is a robust marker for noise-induced synaptopathy in humans. One potential confound is that animal experiments have shown that EFR remains normal as long as there is the capacity to maintain neural gain suggesting that top-down activity, including cognition and memory capabilities, can influence neural responses in the brainstem (Möhrle et al., 2016). These findings suggest that future studies should consider how top-down mechanisms influence the periphery, especially for aged populations. Furthermore, with
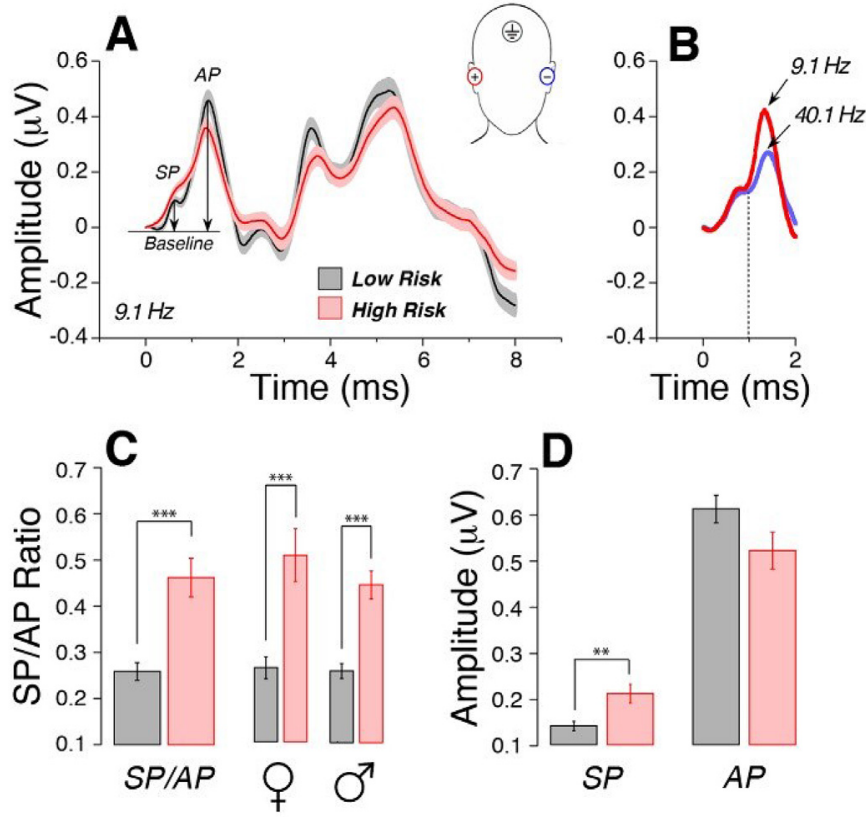

Fig. 3. Evidence of noise exposure-related increase in SP/AP ratio in humans. Electrocochleography shows evidence for cochlear synaptopathy in the high-risk group. A: Averaged waveforms $( \pm$ SEMs) from each group in response to clicks delivered at $9.1 \mathrm{~Hz}$ in alternating polarity at $94.5 \mathrm{~dB} \mathrm{nHL}$. SP and AP are measured from baseline to peak, as illustrated. B: Increasing click rate from $9.1 \mathrm{~Hz}$ to $40.1 \mathrm{~Hz}$ decreases AP without affecting SP: mean waveforms from 6 subjects are shown. C: Mean SP/AP ratio is nearly twice as high in the high-risk vs. the low-risk group. This difference remains when subjects are separated by sex. D: The difference in SP/AP ratios arises from both an increase in the SP and a decrease in the mean AP, although only the SP differences are statistically significant. All data are means $({ } \mathrm{SEM}) .{ }^{* * *} \mathrm{p}<0.001$; ${ }^{* *} \mathrm{p}<0.01$. Reprinted with permission from Liberman et al., 2016, PLoS One.

\section{A}

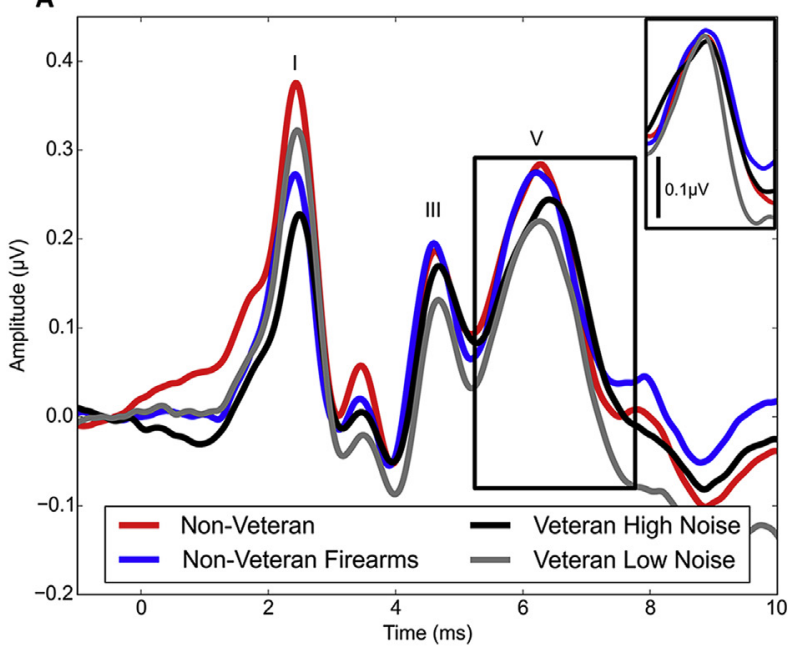

B
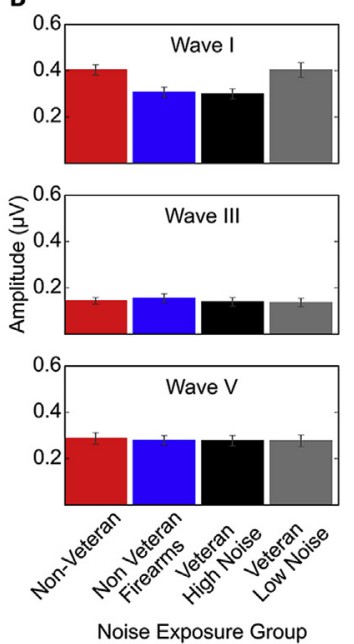

Fig. 2. Evidence of noise exposure-related $A B R$ wave I amplitude reduction in humans.

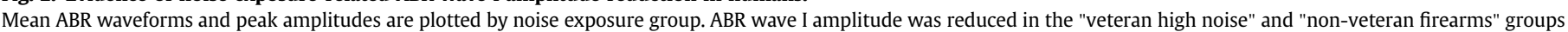

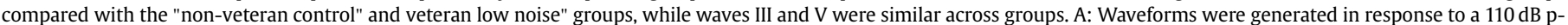

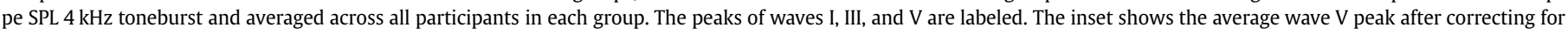

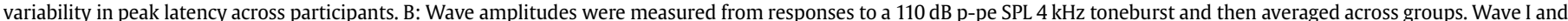

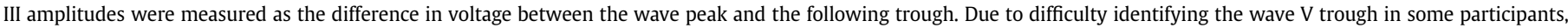

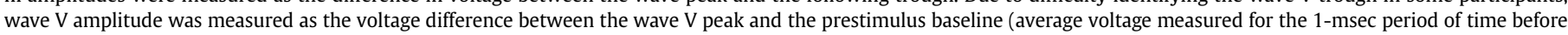

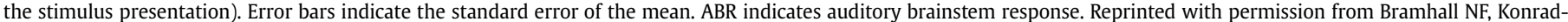

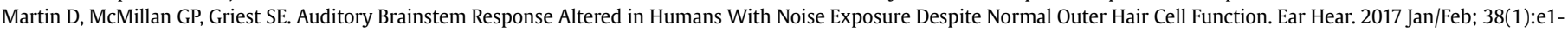
e12. https://insights.ovid.com/pubmed?pmid=27992391. 
better detection tools, other factors that may contribute to impaired neural processing may become feasible to assess, such as potentially detrimental effects of corticosterones and/or potentially beneficial effects of systemic corticosteroids on auditory processing (Singer et al., 2018).

\subsubsection{Data consistent with an impact of synaptopathy on auditory perception}

One advantage of looking at perceptual consequences of synaptic/neural dysfunction is that uncertainties about the reliability of noise exposure questionnaires and their comparability across studies can be taken out of the equation. Instead, a physiological measure (e.g. ABR wave I amplitude, ABR wave I/V amplitude ratio, EFR strength, MEMR strength) can be directly compared to, or correlated with, the perceptual measure.

Tinnitus. Consistent with animal models of cochlear synaptopathy, where ABR wave I amplitude is reduced (Kujawa and Liberman, 2009; Furman et al., 2013; Sergeyenko et al., 2013), several studies have shown a relationship between reduced wave I amplitude (or reduced wave I/V ratio) and tinnitus (Schaette and McAlpine, 2011; Gu et al., 2012; Bramhall et al., 2018; Valderrama et al., 2018). It is not completely clear why the amplitude of wave $\mathrm{V}$ remains close to normal despite the inferred synaptopathy in tinnitus patients, although enhanced central gain after IHC loss is a possible explanation for this phenomenon (for review see Salvi et al., 2016). Decreased responses for two other physiological measures that are sensitive to synaptopathy in mouse models, the MEMR and the EFR (Shaheen et al., 2015; Valero et al. 2016, 2018), have been associated with tinnitus in humans as well. In individuals with tinnitus who have normal or near-normal audiograms, Wojtczak et al. (2017) observed a weakened MEMR relative to age- and sex-matched controls. Paul et al. (2017) showed EFR reductions for individuals with normal audiograms and tinnitus compared to those without tinnitus. However, reanalysis after identification of a statistical error revealed that this was not a significant effect (Roberts et al., 2018).

Hyperacusis. The lack of a uniform measure of hyperacusis makes this perceptual deficit difficult to assess. Bramhall et al. (2018) did not observe a relationship between loudness discomfort level (LDL) and ABR wave I amplitude, but this may be because LDL alone is not a good indicator of hyperacusis (Sheldrake et al., 2015; Zaugg et al., 2016). Liberman et al. (2016) showed that their high noise exposure group was more likely to report annoyance of everyday sounds and avoidance of noisy environments than their low noise exposure group. However, neither their noise exposure nor their hyperacusis questionnaire was validated. In addition, although they showed an increase in SP/AP ratio in their high noise exposure group, they did not specifically analyze the relationship between responses on the hyperacusis questionnaire and ABR measures. Given that the high noise exposure group had significantly poorer extended high frequency (EHF) thresholds than the low noise exposure group, this may account for the increased reporting of sound tolerance problems in the high noise exposure group.

Speech-in-noise performance. Theoretical reasoning predicts that synaptopathy should degrade the neural coding of speech, particularly in noise, and thus hinder the intelligibility of speechin-noise (Lopez-Poveda and Barrios, 2013; Lopez-Poveda, 2014). Liberman et al. (2016) found a relationship between SP/AP ratio and speech-in-noise performance in young males, although the significant differences in the EHF thresholds of the two groups could have impacted speech-in-noise performance (Badri et al., 2011; Yeend et al., 2017). In addition, Prendergast et al. (2018) reported the SP/AP ratio had considerably less test-retest reliability than wave I amplitude within their normal hearing cohort. In a sample that included participants up to age 55 with pure tone thresholds ranging from normal to mild high frequency hearing loss, Valderrama et al. (2018) observed an interaction effect of the ABR wave I/V amplitude ratio and wave V-I interpeak latency on speechin-noise performance, suggesting that slower neural conduction is associated with poorer performance. While average high frequency and EHF auditory thresholds did not appear to be predictive of speech perception performance in this sample, this does not rule out the possibility that subclinical OHC dysfunction may have contributed to the relationship between the ABR metrics and speech perception performance. Although the impact of synaptopathy on speech perception in people with normal audiograms may be limited, it is important to remember that synaptopathy likely co-exists with audiometric loss in many cases of SNHL, and that in the context of OHC dysfunction, synaptic/neuronal loss may have a greater impact on speech perception. This is supported by the findings of Bramhall et al. (2015) showing an interaction effect of average pure tone thresholds (at $0.5,1,2$, and $4 \mathrm{kHz}$ ) and ABR wave I amplitude on performance on the QuickSIN speech intelligibility test, with a stronger relationship between ABR wave I amplitude and QuickSIN performance (poorer performance for lower wave I amplitudes) in individuals with elevated pure tone thresholds. In addition to these peripheral effects, central factors such as attention, working memory and language, are also important factors that affect speech-in-noise performance (Yeend et al., 2017) and likely interact with any peripheral encoding deficits, thus contributing to the wide variation observed in speech-in-noise performance across individuals with similar audiograms (Johannesen et al., 2016; Lopez-Poveda et al., 2017).

Performance on suprathreshold psychoacoustic tasks. Synaptopathy likely degrades the neural coding of acoustic information, particularly in noise (e.g., Lopez-Poveda, 2014). The relationship between auditory encoding of complex stimuli and performance on basic psychoacoustic tasks such as amplitude modulation detection, temporal fine-structure sensitivity, tone-innoise detection, frequency and intensity discrimination, and ITD or interaural level difference (ILD) sensitivity are not well understood. Numerous studies have investigated the relationship between these metrics over the years with mixed outcomes, even for listeners with clinically normal hearing (Strelcyk and Dau, 2009; Hopkins and Moore, 2011; Fullgrabe et al., 2014; Stone and Moore, 2014; Prendergast et al., 2017a; Yeend et al., 2017; Valderrama et al., 2018). Without a better understanding of the relationship between physiological metrics (often representing a population response to a click or AM stimulus) and performance on a psychoacoustic task, we run the risk of comparing apples to oranges, as a single synaptopathy profile may have differing effects on physiological measures versus psychoacoustic tasks. Numerical modelling approaches can improve our ability to compare potential metrics of synaptopathy by predicting the expected impact of synaptopathy and/or OHC/IHC deficits on each measure (Verhulst et al. 2016, 2018b; Encina-Llamas et al., 2017; Paul et al., 2017; Carney, 2018).

Biophysical models of the human auditory periphery have suggested an impact of synaptopathy on the encoding of suprathreshold sounds. Bharadwaj et al. (2014) modelled the effect of a complete loss of low-SR AN fibers on the population response of the inferior colliculus (IC) (a proxy for the dominant source generators of the EFR) (Melcher and Kiang, 1996)) for an $80 \mathrm{~dB}$ SPL 100\% sinusoidally AM pure tone embedded in notched noise and found a $7 \mathrm{~dB}$ reduction in the magnitude of the response. This study did not consider the impact of this reduction on a specific psychoacoustic task, but degraded coding of AM information at the level of the IC is expected to impair performance on a psychoacoustic AM detection task. Verhulst et al. (2018b) expanded on these findings by using a numerical model of the human auditory periphery to compute the 
impact of synaptopathy and OHC loss on the EFR, AM and tone-innoise detection threshold. Complete low-SR fiber loss was predicted to elevate the $4 \mathrm{kHz}$ AM detection threshold by $2 \mathrm{~dB}$ and an additional loss of $50 \%$ or $75 \%$ of the high-SR fiber population resulted in an $A M$ detection threshold shift of $8 \mathrm{~dB}$ and $15 \mathrm{~dB}$, respectively. The simulations also showed that individual differences in AM detection were well correlated to the EFR (in response to a $100 \%$ AM tone) and that synaptopathy, rather than OHC deficits, was the main factor driving individual differences in AM detection performance for listeners with normal audiograms and those with sloping high frequency hearing loss. Furthermore, the simulations predicted the need for a $4 \mathrm{~dB}$ stimulus signal increase for a synaptopathy model (100\% low-SR loss and 50\% high-SR fiber loss) to reach the same performance on a $4 \mathrm{kHz}$ tone-in-noise detection task as a normal hearing model. The simulated tone-in-noise detection differences on the basis of different degrees of synaptopathy were consistent with behavioural chinchilla tone-in-noise detection threshold shifts in the range of 5-10 dB when more than $60 \%$ of the IHC population was lost (Lobarinas et al., 2016). Paul et al. (2017) showed that a simulated loss of low- and medium-SR fibers (based on the Zilany et al. (2014) model) was sufficient to account for individual differences in AM detection thresholds for a $5 \mathrm{kHz}$ pure tone among individuals with normal hearing. In another simulation model, Carney (2018) suggested that synaptopathy may alter spectral contrasts across the cochlear partition, which could impair encoding of speech. These studies suggest that model simulations are a promising method for disentangling the role of different AN fiber populations on suprathreshold auditory perception as well as the interaction between synaptopathy and $\mathrm{OHC} / \mathrm{IHC}$ dysfunction.

\subsection{Data inconsistent with noise-induced synaptopathy in humans}

As outlined earlier, there are two basic approaches to the search for noise-induced cochlear synaptopathy in humans. The first is to determine if noise exposure is associated with neural deficits (e.g., wave I amplitude changes) consistent with those observed in animals with histologically confirmed synaptopathy. The second is to determine whether or not noise exposure (with or without changes in evoked potential measurements) is associated with a measurable change in auditory function (difficulties understanding speech in complex listening situations).

A number of studies have used the first approach, specifically seeking evidence of neural deficits that parallel those observed in rodent models. To reduce the probability of inclusion of participants with significant $\mathrm{OHC}$ loss, human studies have largely recruited listeners with hearing thresholds within the clinically normal range ( $\leq 20 \mathrm{~dB} \mathrm{HL}$ ) and measured the amplitude of wave I of the ABR, the measure of AN function that is associated with synaptopathy in rodent models (Kujawa and Liberman, 2009; Sergeyenko et al., 2013). It needs to be mentioned that there are several morphological differences between rodent and human auditory neurons that could explain the difficulty in detecting synaptopathy in humans. For example, the total number of spiral ganglion cells and AN fibers differ and in contrast to rodents, nerve fibers in humans are rarely myelinated (Kimura et al., 1979; Nadol Jr, 1988).

The majority of studies have failed to find a significant relation between questionnaire- or interview-based estimates of noise exposure and wave I amplitude among participants with normal audiometric hearing (Fulbright et al., 2017; Grinn et al., 2017; Guest et al., 2017b; Prendergast et al. 2017a, 2018; Spankovich et al., 2017; Johannesen et al., 2019) (Figs. 4 and 5). The largest study to date (126 participants) used a comprehensive lifetime noise interview to estimate noise exposure history but failed to detect significant
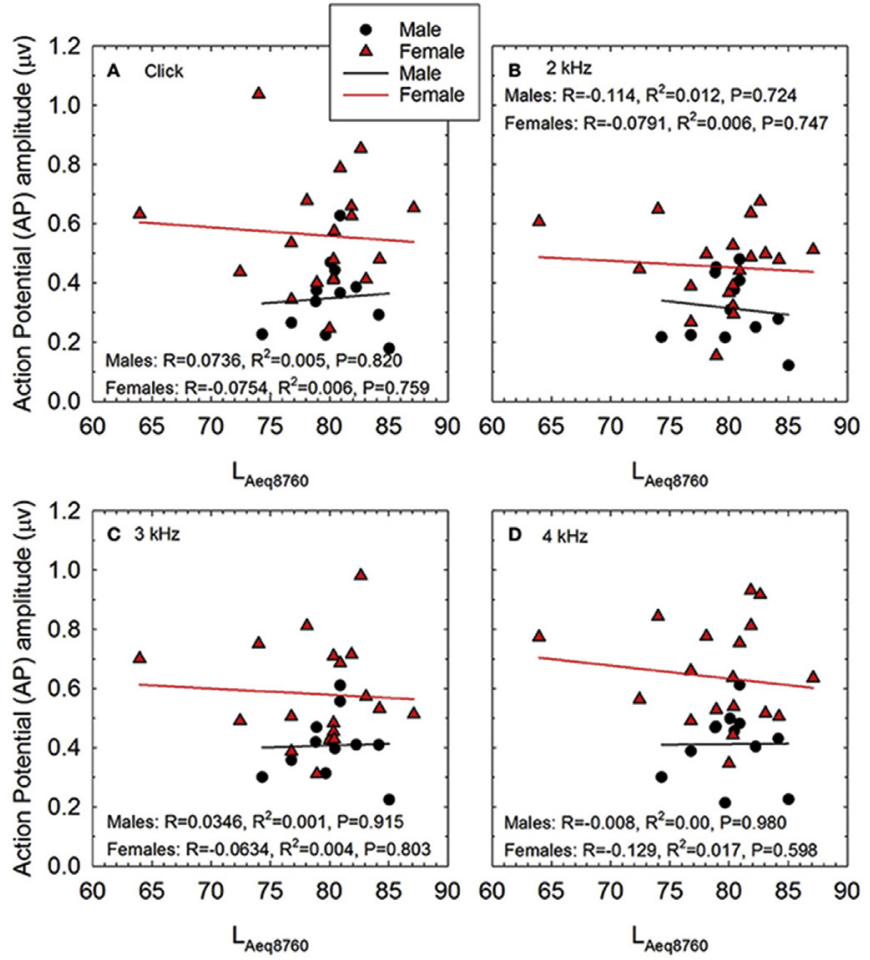

Fig. 4. Evidence that self-reported noise exposure is not correlated with ABR wave I amplitude in humans.

The relationship between self-reported noise exposure (calculated as $\mathrm{L}_{\text {Aeq8760 }}$ ) and action potential (AP) amplitude is shown for male and female participants for stimuli including A: clicks, B: $2 \mathrm{kHz}$ tone bursts, C: $3 \mathrm{kHz}$ tone bursts, and D: $4 \mathrm{kHz}$ tone bursts. All AP amplitude data were normally distributed. Pearson correlation analysis revealed no statistically significant relationships between self-reported noise history and AP amplitude within males or females. Lines of best fit are shown (Males: black symbols and regression lines; Females: red symbols and regression lines). Reprinted with permission from Grinn et al. (2017), Frontiers in Neuroscience. (For interpretation of the references to colour in this figure legend, the reader is referred to the Web version of this article.)

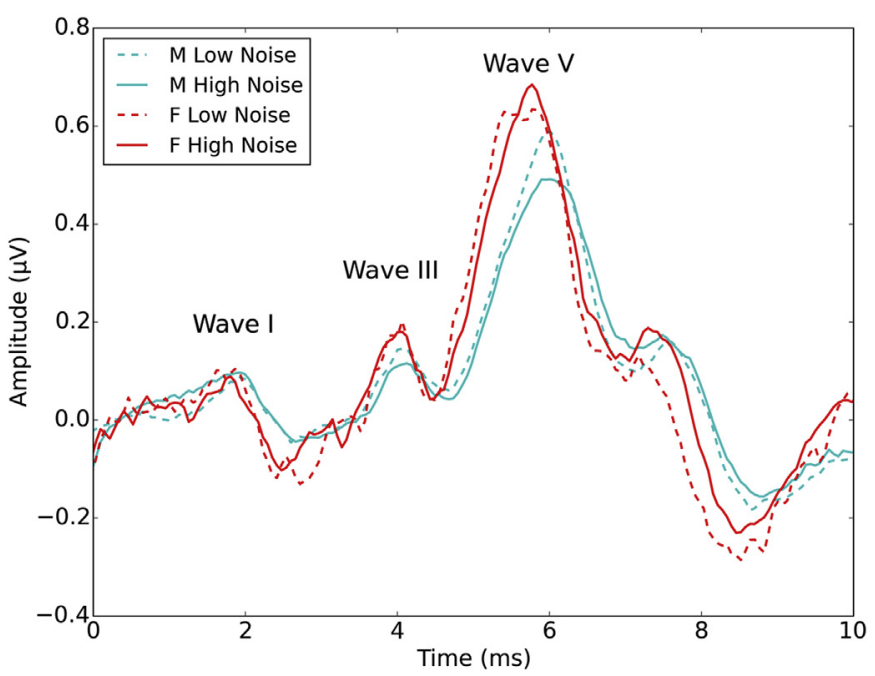

Fig. 5. Evidence that $A B R$ wave $I$ amplitude is not decreased by noise exposure in humans.

Grand average ABR waveforms. Average waveforms are shown in microvolts for males and females separately and for the 15 lowest and 15 highest noise exposed individuals for each sex. Waves I, III and V can be seen at around 2, 4 and $6 \mathrm{~ms}$ respectively. Waveforms are plotted broadband in order to show the full morphology of the response. Reprinted with permission from Prendergast et al. (2017), Hearing Research. 
decreases in wave I amplitude with increasing noise exposure, despite the presence of EHF hearing loss (Prendergast et al., 2017a), raising questions about the prevalence of this pathology in humans with "typical" noise histories. Also of interest is the study of Fulbright et al. (2017), who collected data using the same methodology and stimulus conditions as the earlier study by Stamper and Johnson (2015a). A reanalysis of this earlier study by sex reported a significant correlation between noise exposure and wave I for female participants, but not males (Stamper and Johnson, 2015b). However, when Fulbright et al. added their data to those of Stamper and Johnson, the effect was no longer significant for females either, suggesting that this original result may have been a statistical fluke. Several other groups have used the noise exposure questionnaire (NEQ) as used by Stamper and Johnson, without finding statistically significant relationships between NEQ scores and wave I amplitude (Grinn et al., 2017; Spankovich et al., 2017; Ridley et al., 2018). Skoe and Tufts (2018) did not detect differences in wave I amplitude, although they did report delayed latencies of waves I through $\mathrm{V}$, with increasing delays for later waves. In this study, the participants were divided into low- and high-exposure groups based on noise dosimetry over a one-week period.

As indicated earlier, another potential electrophysiological measure of synaptopathy that has received attention is the EFR, which has been suggested as a sensitive measure of low-SR fiber loss, especially at high stimulus levels and shallow modulation depths (Bharadwaj et al. 2014, 2015). Again, however, the evidence for an association with noise exposure is weak. Prendergast et al. (2017a), Guest et al. (2017b), and Grose et al. (2017) have all reported no significant relation between lifetime noise exposure and EFR amplitude.

\subsubsection{Studies failing to find evidence that noise-induced cochlear synaptopathy is functionally significant in humans}

The second basic approach taken in studies of noise-induced cochlear synaptopathy in humans is to determine whether or not noise exposure (with or without changes in evoked potential measurements) is associated with a measurable change in auditory function. Recent studies that have taken this approach have provided little evidence that noise exposure is related to perceptual deficits for listeners with normal audiometric hearing. In a study of 138 participants aged 18-36 with clinically normal hearing, Prendergast et al. (2017b) reported little relation between lifetime noise exposure and a range of perceptual measures, including frequency discrimination, intensity discrimination, interaural phase discrimination, AM detection, auditory localisation, musical consonance perception, and speech perception in noise (SPiN). Similarly, in an older cohort of 122 participants aged 30-57, Yeend et al. (2017) reported no relation between lifetime noise exposure and a range of auditory processing and SPiN tasks. Le Prell et al. (2018) similarly failed to detect any statistically significant relations between common sources of noise exposure and performance on a word-in-noise test. These null results are consistent with several other studies (Fulbright et al., 2017; Grinn et al., 2017; Grose et al., 2017; Guest et al., 2018).

Another approach that avoids issues with the unreliability of self-reported noise exposure is to determine whether or not perceptual deficits are associated with physiological measures assumed to reflect cochlear synaptopathy. Several recent studies have reported no relation between ABR wave I amplitude and SPiN (Fulbright et al., 2017; Grinn et al., 2017; Prendergast et al., 2017b; Bramhall et al., 2018; Guest et al., 2018), nor between EFR amplitude and SPiN (Prendergast et al., 2017b; Guest et al., 2018) (Fig. 6). With regard to tinnitus, Gilles et al. (2016), Guest et al. (2017a; 2017b), and Shim et al. (2017), have each reported no relation between presence of tinnitus and wave I amplitude for participants

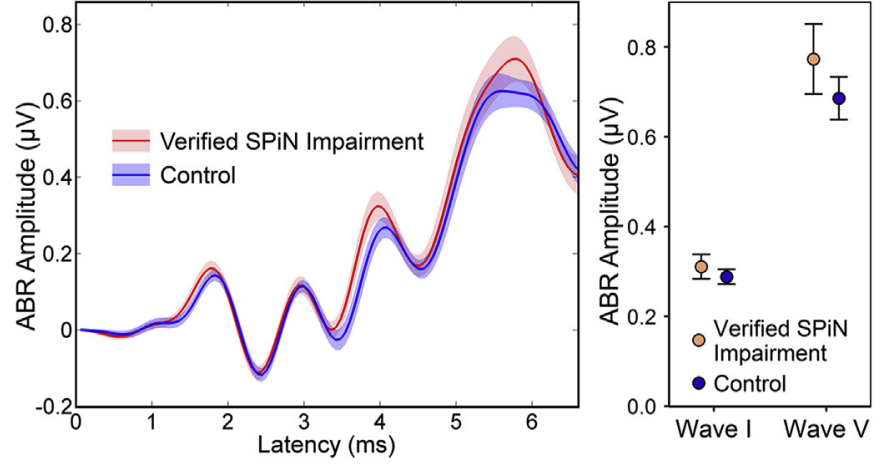

Fig. 6. Evidence that ABR wave I amplitude is not decreased among individuals with problems understanding speech-in-noise.

ABRs elicited by $102 \mathrm{~dB}$ peSPL clicks for verified-SPiN-impairment and control groups. A: Grand average waveforms (averaged across ears and across participants). Shaded areas represent the SEM. B: Wave I and wave $\mathrm{V}$ amplitudes, presented as mean $\pm \mathrm{SEM}$. Reprinted with permission from Guest et al., 2018, Hearing Research.

with normal audiometric hearing. Guest et al. (2017a; 2017b) also reported no significant reduction in EFR amplitude in their tinnitus participants compared to controls.

\subsection{Possible explanations for null results and differences between studies}

It is invalid to assume that a non-significant result implies that the null hypothesis has been proven. The following comments offer potential reasons for null results and differences in outcomes across studies. Many of the issues noted below have the net effect of reducing statistical power, as they introduce variability into the data.

\subsubsection{Humans may be less vulnerable to noise-induced} synaptopathy than rodents

Cochlear synaptopathy is observed with $100 \mathrm{~dB}$ SPL 2-h octave band exposures in the mouse (Kujawa and Liberman, 2009), $\sim 106 \mathrm{~dB}$ SPL 2-h octave band exposures in the guinea pig (Lin et al., 2011), and $\sim 109 \mathrm{~dB}$ SPL 2-h octave band exposures in the rat (Lobarinas et al., 2017). Decreasing sound levels by $3 \mathrm{~dB}$ can eliminate synaptopathic injury (see Fernandez et al., 2015), whereas increasing sound levels by $3 \mathrm{~dB}$ can intensify the injury to include permanent threshold shift (Lin et al., 2011). Macaque monkeys are more resistant to cochlear synaptopathy than rodents (Valero et al., 2017), resulting in predictions that humans are less susceptible to noise-induced synaptopathy than rodents (Dobie and Humes, 2017). Given the high intensity levels needed to produce acoustic trauma resulting in significant temporary threshold shift, bordering on a permanent threshold shift, there may be few human exposures that will result in the large reductions in ABR wave I seen in the original mouse study (Kujawa and Liberman, 2009). If so, this would make selective noise-induced cochlear synaptopathy harder to detect in humans. Indeed, when Dobie and Humes adjusted for inter-species differences in susceptibility to noise-induced temporary threshold shift, they found that the noise exposures that cause neuropathy in rodents, when translated to the equivalent levels predicted to be needed to induce cochlear synaptopathy in humans, exceed the OSHA permissible exposure limits. This suggests that the noise exposure levels that are synaptopathic for humans may already be addressed by current noise exposure guidelines; additional research using repeated exposure paradigms is urgently needed to better model occupational risk (for recent review, see Le Prell, 2019). 
1.3.2. The range of exposures inducing selective cochlear synaptopathy may be narrow

In a recent macaque study, noise exposures producing a temporary threshold shift were associated with only a $12-27 \%$ loss of synapses (Valero et al., 2017) versus $40-55 \%$ loss in rodent models (Kujawa and Liberman, 2009; Lin et al., 2011; Hickox et al., 2017). Given that primates appear more resistant to noise-induced synaptopathy than mice (Kujawa and Liberman, 2009; Valero et al., 2017), there may only be a narrow "sweet spot" where noiseinduced cochlear synaptopathy can occur while hearing thresholds are still clinically normal. This sweet spot would be characterized by sufficient synaptopathy to be detectable via ABR amplitude measurements (or another less variable, more reliable metric), but with overall cochlear damage low enough that OHCs are intact and hearing thresholds are normal. It is possible that this "sweet spot" is often the result of a combination of noise- and agerelated synaptopathy. Support for this suggestion comes from the observation that several studies investigating young people with recreational noise exposure or tinnitus have failed to find evidence for synaptopathy in ABR wave I amplitude measurements (Fulbright et al., 2017; Grinn et al., 2017; Guest et al. 2017b, 2018; Prendergast et al., 2017a), whereas studies in slightly older cohorts (Schaette and McAlpine, 2011; Gu et al., 2012; Valderrama et al., 2018) did find reductions in ABR wave I amplitude in the experimental group. There is also the possibility that partial synaptic repair may occur in humans following noise exposure. This phenomenon has been observed in noise-exposed guinea pigs (Liu et al., 2012; Shi et al., 2016) and if also prevalent in humans, it would be yet another source of variation impacting our ability to find evidence for synaptopathy.

\subsubsection{Sound-evoked potentials are more variable in humans than in rodents}

In the study of Prendergast et al. (2018), the coefficient of variation in wave I amplitude was $25 \%$ in the low noise exposure group, which may indicate a large degree of variability compared to the effect being measured. One of the factors that may contribute to the between-subject variability and reduced statistical power for detection of differences in human electrophysiological measures is head size and geometry (Mitchell et al., 1989; Don et al., 1994); this may contribute to differences in the average ABR wave I amplitude for males and females, with smaller average wave I amplitudes in males than in females. Cochlear duct length also varies with sex, with longer duct length in males than in females (Sato et al., 1991; Thong et al., 2017). The higher noise floor of human ABR wave I amplitude measurements is another potential source of variability. Humans are tested while unanesthetized (with a variable sleep state) and with dermal or ear canal electrodes, while rodents are tested while anesthetized using subcutaneous needle electrodes. An additional aspect that needs further investigation is the possibility that top-down regulation might be playing a role.

\subsubsection{The sensitivity of the auditory brainstem response to human synaptopathy might be inadequate}

Most human studies have employed ABR amplitude measurements to assess cochlear synaptopathy (Schaette and McAlpine, 2011; Gu et al., 2012; Stamper and Johnson, 2015a; Liberman et al., 2016; Bramhall et al. 2017, 2018; Fulbright et al., 2017; Grinn et al., 2017; Grose et al., 2017; Guest et al. 2017b, 2018; Prendergast et al., 2017a; Shim et al., 2017; Valderrama et al., 2018). In these studies, a decrease in the amplitude of $A B R$ wave I relative to wave $\mathrm{V}$ has been interpreted as evidence for cochlear synaptopathy, based on speculation that wave V amplitude is "normal" as a consequence of the compensatory central gain observed in animal models (see Salvi et al., 2017). The interpretation of wave I/V ratios must be considered hypothetical at this time, as central gain as a compensatory mechanism subsequent to the loss of synapses in the cochlea is highly speculative and not well understood. It is also possible that not all cases of synaptopathy lead to increased central gain. Using ABR wave I amplitude as an indicator of synaptopathy is further complicated by the fact that high frequency $\mathrm{OHC}$ loss also reduces $A B R$ wave I amplitude by decreasing the contribution of high frequency AN fibers to the ABR generation (e.g., Lewis et al., 2015; Verhulst et al., 2016). In addition, it must be remembered that the OHCs provide significant level-dependent amplification of the cochlear response, and loss of the OHCs decreases the input to the IHCs (Dallos et al., 2006; for recent review see Le Prell, 2019). This makes it difficult to use ABR wave I amplitude to diagnose synaptopathy when OHC dysfunction is also present. Thus, synaptopathy might remain "hidden" even if ABR amplitude measurements are added to the audiometric test battery.

In addition, the results of Bourien et al. (2014) suggest that ABR wave I amplitudes might not be a particularly sensitive measure of low-SR synaptopathy. In a series of measurements in gerbils, Bourien et al. showed that low-SR AN fibers have a minimal contribution to the amplitude of ABR wave 1 . There are, however, indications, that low-SR fibers might be more important in controlling the efferent system than in encoding of high-intensity sound levels (Carney, 2018). This is also consistent with the modelling work in Encina-Llamas et al. (2017) where the EFR is dominated by off-frequency high-SR fibers. Removing all low-SR fibers shows hardly any contribution in a model based on AN responses. Interestingly, when Furman et al. (2013) demonstrated particular vulnerability of low-SR fibers to synaptopathy, they binned the low- and medium-SR fibers together in their analysis. Therefore, the possibility for a significant contribution of mediumand high-SR fiber loss to synaptopathy (and ABR wave I amplitude) should also be considered.

\subsubsection{The sensitivity of the envelope following response to human synaptopathy might be inadequate}

In addition to the concerns noted above, there are other factors that suggest the second main electrophysiological measure of synaptopathy in rodents, EFR amplitude, may also be insensitive to synaptopathy in humans, consistent with the lack of a clear reduction in EFR strength in individuals with a history of noise exposure. Modelling of AN activity suggests that low-SR fibers have limited contribution to the EFR at high stimulus levels and that amplitude fluctuations in the stimulus are coded by the activity of high-SR fibers at frequencies basal to the frequency of the stimulus (Encina-Llamas et al., 2017). Accordingly, low-SR fiber loss will not impact the EFR due to the large population of high-SR fibers contributing to the response. Empirical data will be necessary to resolve these questions, given discrepancies in predictions across the various modelling efforts. Furthermore, as noted earlier, in the mouse model, the EFR is sensitive to synaptopathy at high modulation rates (around $1 \mathrm{kHz}$, Shaheen et al. (2015)) but does not seem to be sensitive to synaptopathy at the lower rates (typically $100 \mathrm{~Hz}$ ) used in the human studies.

\subsubsection{Variability in the noise-exposed populations studied could underlie observed differences in results and conclusions}

Most studies of noise-induced synaptopathy have investigated young people with clinically normal hearing and high versus low recreational noise exposure (e.g. concerts, personal music player use, etc.). Many of these studies have not found an effect of noise exposure on suprathreshold ABR wave I amplitude, either using the noise exposure survey scores as a continuous variable or when participants are sorted into high and low exposure groups using survey data (Fulbright et al., 2017; Grinn et al., 2017; Prendergast 
et al., 2017a). Similarly, Holtegaard \& Epp (2018) found no difference in ABR wave I amplitude for individuals with a history of occupational noise exposure (musicians and flight attendants) compared to controls with less reported noise exposure. In contrast, studies of young music students (Liberman et al. (2016) and young female adults (Stamper and Johnson, 2015a; 2015b) have found electrophysiological differences as a function of noise exposure history, consistent with synaptopathy. The few studies that have included older participants or individuals with higher levels of noise exposure have found noise exposure-related reductions in ABR wave I amplitude (Bramhall et al., 2017; Valderrama et al., 2018). Common recreational exposures and many occupational exposures are very different from the high-intensity military noise and firearms to which Bramhall et al.'s participants were exposed. If it is the case that humans are not as susceptible as rodents to noise-induced synaptopathy (Dobie and Humes, 2017; Valero et al., 2017), then it is likely that the intensity levels of many common sources of recreational noise exposure are simply not high enough to cause synaptopathy.

\subsubsection{Differences in $\mathrm{OHC}$ function between control and} experimental groups could confound results and interpretation

Even among young people with normal audiograms, subclinical $\mathrm{OHC}$ dysfunction is more likely in those with noise exposure than those without. This could affect electrophysiological and perceptual measures, leading to between-group differences that are not solely related to synaptopathy.

\subsubsection{Variability in the tinnitus populations used across studies} could underlie observed differences in results and conclusions

Recruitment strategies across studies investigating ABR wave I amplitude and tinnitus have varied (Schaette and McAlpine, 2011; Gu et al., 2012; Guest et al., 2017b; Shim et al., 2017; Bramhall et al., 2018), which may contribute to the differing results. Tinnitus is a heterogeneous disorder with noise exposure as one possible cause. Other etiologies of tinnitus include head/neck trauma, medications, thyroid problems, cardiovascular disease, acoustic neuroma, Meniere's disease, etc. (Henry et al., 2014). When subjects are recruited specifically based on their report of tinnitus, it is expected that there will be a mix of underlying etiologies for the tinnitus. However, if a study recruits for noise exposure and then looks at the subgroup of noise-exposed participants that have tinnitus, that tinnitus group is more likely to have predominantly noise-induced tinnitus.

\subsubsection{Functional metrics are variable; some tests may not have adequate sensitivity or specificity}

It is also perhaps unsurprising that it has proven difficult to find evidence that synaptopathy leads to deficits in behavioural performance. Oxenham (2016) has argued, from a signal detection theory perspective, that the effects of even $50 \%$ deafferentation may be insignificant perceptually. Other perceptual (Lopez-Poveda and Barrios, 2013; Marmel et al., 2015) and computational models, however, suggest larger effects (4-7 dB) (Paul et al., 2017; Verhulst et al., 2018b) depending on the stimulus characteristics and the amount of deafferentation. Empirical data are needed to assess these varied model predictions. However, the models recently described by Carney (2018) suggest an even more fundamental paradigm change may be necessary, arguing against a direct role of low- and medium-SR fibers in coding sounds at moderate to high sound levels.

Many auditory and non-auditory factors, such as memory and attention, are known to contribute to behavioural tasks such as SPiN (Yeend et al., 2017), and the contribution of synaptopathy may be relatively small, at least for listeners with clinically normal audiograms. Additional research is needed to determine the relative contributions of $\mathrm{OHC}$ function, cochlear synaptopathy, memory, attention, and other factors on auditory perception. If associations between cochlear synaptopathy and perceptual issues cannot ultimately be reliably measured, even in individuals with significant synaptic loss, such results would raise questions as to whether noise-induced cochlear synaptopathy should be regarded as a major hearing health issue. While cross-sectional retrospective study designs are useful and powerful, longitudinal studies allowing the trajectory of change to be established in parallel across a detailed test battery would be helpful in more completely answering these questions.

\subsubsection{Noise exposure history metrics are variable and imprecise} exposure measurements introduce variability

Different groups have used a variety of measures of selfreported noise exposure history in their studies of noise-induced synaptopathy (Bharadwaj et al., 2015; Stamper and Johnson, 2015a; Liberman et al., 2016; Bramhall et al., 2017; Grinn et al., 2017; Grose et al., 2017; Paul et al., 2017; Prendergast et al., 2017a; Yeend et al., 2017; Holtegaard and Epp, 2018). Although synaptopathy can theoretically be induced by noise exposure experienced at any point in an individual's lifetime, some of these metrics assess noise exposure only during the previous year or two rather than over their lifetime. Although participants were excluded if they reported that the previous year was not representative of historic exposure, surveys based on the previous year have not been validated against lifetime surveys. Except for Bramhall et al. and Yeend et al., these noise exposure measures either do not specifically ask about firearm use or they do not incorporate firearm exposure into the overall noise exposure score because they use a scoring system that does not allow for both continuous and impulse/impact noise exposures. However, this is probably not a significant confound for the European studies, where firearm use is minimal. In addition, all self-report measures are dependent on the recall ability of the participants. This makes noise exposure history questionnaires a relatively crude metric that is prone to measurement error. There is no consensus on which noise exposure questionnaire should be used for studying synaptopathy or how to score it, making comparisons across studies difficult.

It has been argued that the imprecision of the self-report noise exposure metrics is small compared to the range of noise exposures in the sample of participants used in some studies. For example, in one study reporting a null result for ABR wave I, the low- and highexposure groups differed by an average of a factor of 340 in terms of estimated lifetime energy of exposure (Prendergast et al., 2018). The mean exposure for the low-noise group in this study was equivalent, in terms of total energy, to that for an individual who goes to a nightclub or live music event for $1.5 \mathrm{~h}$, once per year, for 5 years. The mean high-noise exposure was equivalent to going to the same event for $3 \mathrm{~h}$, three times per week, every week of the year, for 5 years. It seems unlikely that participants' recollection of exposures would be so poor as to be unable to distinguish between these. Furthermore, the lifetime noise-exposure measure used by Prendergast, Guest, and colleagues, was significantly correlated with 16-kHz thresholds (Prendergast et al., 2017a) and with the presence of tinnitus (Guest et al., 2017b), suggesting that this measure is reliable. However, measurement error in estimates of noise exposure remains a significant concern. The potential for errors associated with the assignment of sound intensity levels to recreational exposures is highlighted by Le Prell et al. (2018), who measured preferred music player listening levels across multiple songs per subject. Even within a quiet lab setting, individual subjects had significant variability in their level selections on a song- 
to-song basis. These data raise questions about the validity of assigning a relatively arbitrary intensity level for calculating accumulated noise exposure over the past year or longer periods of time. Differences in accumulated noise dose are highly variable across events and individuals, as a function of differences in event intensity level, distance from the sound source, and duration of event attendance (see for example, the event-specific exposure data in Grinn et al. (2017)).

\subsubsection{Control groups may differ across studies}

In group comparisons, it is vital that the control population has limited noise exposure, otherwise the presence of people with synaptopathy in the control group will make it difficult to detect differences between the control and experimental groups. Ensuring a control group with limited noise exposure is difficult due to the inherent limitations of using noise exposure questionnaires. An indepth noise exposure questionnaire with specific questions about a variety of potentially noisy activities rather than a questionnaire that uses more general questions to assess noise exposure history may be necessary to aid recall of noise exposures in potential study participants, particularly for infrequent exposures. Given the results from Bramhall et al. (2017) suggesting that firearm users have reduced $A B R$ wave I amplitudes, even individuals with a single episode of firearm exposure should not be included in a control group. Confirming good $\mathrm{OHC}$ function by screening for otoacoustic emissions (OAEs) and/or EHF thresholds will also help ensure this population does not have noise exposure history that they have forgotten to report. It is also possible that most adult humans have some degree of age-related and/or noise-induced synaptopathy, making it difficult to identify a true control population, and obscuring variation between groups.

\subsubsection{Positive results may be due to audiometric confounds unrelated to synaptopathy}

Several studies have provided intriguing evidence that could support an interpretation of an underlying synaptopathic injury. However, there are potential confounds in some of these studies that should be considered. Some studies that have reported a relation between ABR measures of synaptopathy and noise exposure have either reported high-frequency audiometric differences between low- and high-noise groups (Liberman et al., 2016; Bramhall et al., 2017), or have not measured audiometric thresholds at extended high frequencies above $8 \mathrm{kHz}$ (Stamper and Johnson, 2015a; 2015b). Bramhall et al. (2017) reported a 2-6 kHz threshold elevation compared to controls for one of their highnoise groups ("veteran high noise"), but not the other ("non-veteran firearms"). However, they controlled for variability in OHC function in their analysis by statistically adjusting for DPOAE differences between the groups. It is unclear the extent to which small audiometric differences might influence the electrophysiological measures of synaptopathy, although it is known that ABR wave I amplitude is dependent on basal cochlear generators (Don and Eggermont, 1978). Valderrama et al. (2018) reported a weak but significant relation between lifetime noise exposure and ABR wave I amplitude, even after controlling for audiometric thresholds. Although this was a relatively large sample with careful documentation of lifetime exposure to noise, the authors note that if a single outlier with extremely low noise and an extremely robust ABR wave I amplitude was excluded from the analysis, the observed association between lifetime noise exposure and ABR wave I amplitude was no longer statistically significant.

Some of the positive findings with respect to tinnitus and synaptopathy may also have been affected by audiometric differences. In the Gu et al. (2012) study the groups were not audiometrically matched for the click level (120 dB peSPL) at which a significant effect on wave I amplitude was observed, with higher thresholds in the tinnitus group at frequencies of $8 \mathrm{kHz}$ and above. In the Bramhall et al. (2018) study there were also audiometric differences between the groups, although the authors controlled for DPOAE differences in the analyses. In the Schaette and McAlpine (2011) study there was a small audiometric threshold elevation (3.5 dB) in the tinnitus group at $12 \mathrm{kHz}$, and thresholds at higher frequencies were not reported. Wojtczak et al. (2017), who reported a large reduction in the acoustic MEMR amplitude in their tinnitus participants compared to controls, also observed substantial audiometric differences between groups. Although the effect of group was still highly significant after controlling for audiometric threshold, the pure tone threshold measurements were limited to a minimum of $0 \mathrm{~dB} \mathrm{HL}$, which may have biased thresholds for the controls upwards. It is unclear, however, if this could account for the large group differences in MEMR amplitude they observed.

\subsubsection{Reproducibility is a major concern}

Finally, we should be aware of the crisis in the wider neuroscience community regarding reproducibility (Colquhoun, 2017). Many of the human studies of synaptopathy have used a large number of outcome measures. Studies have often reported positive effects for one measure but not others, and the statistical significance of the positive effects has often been marginal (and usually uncorrected for multiple comparisons). In these circumstances, the risk of statistical Type I errors is very high.

\section{Suggestions for methodological approaches to investigate synaptopathy in humans}

With the current state of technology, synaptopathy is a pathology that can only reliably be revealed using histological techniques post-mortem. Because between-study differences in results may be due to methodological differences, researchers around the world are working to identify the "best" (most sensitive) noninvasive measures for detecting synaptopathy in humans. Ultimately, a test battery should be sensitive to synaptopathy both when auditory thresholds are normal, as well as when other auditory deficits are present. However, given that most studies of synaptopathy in humans have used samples with clinically normal or near normal hearing thresholds, it is difficult to recommend the best test measures for diagnosing synaptopathy in individuals with abnormal auditory thresholds. Therefore, the following recommendations are oriented towards diagnosis of synaptopathy in people with normal audiograms. Many of the essential components of the test battery may be necessary in order to have confidence in inferences regarding synaptopathy. The recommendations are as follows:

o Noise exposure measurement tools: At this time, a variety of retrospective self-report tools are being used to investigate noise-induced synaptopathy. Some are survey based and emphasize the past year; others are interview based and emphasize lifetime noise exposure history. The strongest approach would include prospective monitoring of changes in the auditory measures described below as a function of noise exposure documented via dosimetry, but such data will be difficult to collect over an individual's lifetime. The more practical goal should be the development of standardized survey tools that can be used across laboratories, although these are inevitably subject to recall bias. An alternative approach is the recruitment of subjects with specific risk factors (e.g. frequent exposure to very high-intensity amplified music, exposure to firearm discharge, work in a high-level noise environment) with 
planned comparisons with lower-exposure control subjects matched for age and sex.

o Otoscopy: inspection of the ear canals is necessary to exclude participants with potential obstruction of the ear canal or other pathology that may confound the results.

o Tympanometry: measurement of ear drum mobility while the pressure in the sealed ear canal is systematically changed; this is necessary to document a correctly functioning middle ear system.

o DPOAEs: a measure of OHC function, necessary for differential allocation of deficits to $\mathrm{OHC}$ or AN damage. Note that if sound conduction through the middle ear is compromised, DPOAEs will be reduced or absent even if the $\mathrm{OHC}$ population and function are intact.

- During screening tests, DPOAEs are often scored as pass/fail based on whether their levels are at least $6 \mathrm{~dB}$ above the noise floor. This is inadequate and more stringent criteria should be used to guarantee normal $\mathrm{OHC}$ function.

- When DPOAEs are used diagnostically, they are more commonly defined as present and normal, present but abnormal, or absent, with present but abnormal used to identify DPOAE responses that are present but at a reduced amplitude. Empirical research is necessary to identify whether use of these three categories has adequate specificity and sensitivity for sorting participants in these studies.

- DPOAE testing with $f_{1}$ and $f_{2}$ primary tone levels of $65 \mathrm{~dB}$ SPL and $55 \mathrm{~dB}$ SPL are common. A DP-gram obtained at these stimulus levels can be compared to normative values (Gorga et al., 1997, Table A1). Restricting study participation to individuals with DPOAE levels above the 95th percentile for Gorga et al.'s impaired sample will greatly limit OHC dysfunction. However, in noise-exposed samples, this may make it difficult to meet recruitment targets.

- Testing at lower SPLs should also be considered; noiseinduced deficits may emerge at lower SPLs prior to higher SPLs and thus subtle changes in OHC function can be missed.

- As the availability of clinical equipment capable of measuring high-frequency DPOAEs continues to improve, the ability to adjust for OAE amplitude may continue to improve, and it may be advisable to require "normal" OAEs of all study participants at all tested frequencies (Bramhall et al., 2017). Although inclusion of OAEs might improve the ability to interpret study outcomes, it must also be remembered that normal DPOAEs can be recorded even in the presence of $\mathrm{OHC}$ damage and thus normal DPOAEs do not necessarily imply the OHC population is not damaged (Subramaniam et al. 1994a, 1994b; Chen and Fechter, 2003). In addition, OAEs are not sensitive to IHC function, and therefore controlling for OAEs will not guarantee perfect matching between groups.

o Pure tone air conduction thresholds, including EHF assessment: Conventional threshold assessment is necessary, including 3 and $6 \mathrm{kHz}$, and it is essential that EHF assessment be completed up to $12-16 \mathrm{kHz}$. Multiple studies have provided evidence of deficits in the high frequency range related to noise exposure history, with or without corresponding changes in ABR wave I amplitude. As described earlier, ABR wave I is sensitive to basal cochlear function, so it may be important to control for EHF thresholds when making comparisons between participants using this metric.

o ABR: a measure of the sound-evoked neural response, evoked by tones or clicks. Protocols vary significantly across laboratories; in the absence of more sensitive metrics, this is the current gold standard in animal models and should be included in human studies. o Clicks will activate larger regions of the cochlea than tones; some laboratories record responses to both clicks and tones while others only report responses to clicks. In order to reduce the potential impact of $\mathrm{OHC}$ loss in subjects with EHF hearing loss, low-pass filtered clicks could be used. At high intensities, ANFs at high CFs will only contribute through their tails, which are not affected by OHC loss. To facilitate comparisons across studies, clicks should be included in all investigations, and tones, chirps, and other shaped signals should be considered as optional additions. Because the original data from Kujawa and Liberman (2009) reveal frequency-specific effects with both more cochlear synaptopathy in basal regions and greater wave I deficits at higher frequencies, it is reasonable to predict that non-click signals may provide insight into patterns of damage within the human cochlea but we do not yet have sufficient evidence to recommend specific protocols. If data emerge documenting increased sensitivity with non-click signals, these recommendations should be reevaluated.

o In general, click levels vary from as low as $70 \mathrm{~dB}$ nHL to as high as $100 \mathrm{~dB}$ nHL. Some groups report these stimulus levels in $\mathrm{dB}$ $\mathrm{nHL}$, while others report them in $\mathrm{dB}$ peSPL. To facilitate comparisons across studies, both $\mathrm{dB}$ nHL and $\mathrm{dB}$ peSPL should be included in all reports. Based on both animal data and the studies reporting ABR wave I deficits consistent with synaptopathy, 90 and $100 \mathrm{~dB}$ peSPL stimuli are likely to be the most sensitive in revealing wave I deficits; at least one of these higher-level conditions should be included.

o Most human studies consider click durations of 80-100 $\mu \mathrm{s}$ (see Table 1) to characterize the onset response of the population of AN fibers. It should be noted that adopting longer duration click or tone-burst stimuli with different windowing properties are known to alter the frequency-dependent sources which contribute to the ABR amplitude (Rasetshwane et al., 2013). The exact stimulus specifics for the ABR might thus also have an impact on their sensitivity to synaptopathy and/or on the AN fibers types which contribute to the population response.

o Responses may be measured using dermal electrodes or ear canal electrodes; ear canal electrodes are increasingly used in more recent studies to improve resolution of wave I.

o Overall configuration for recordings may be a one-channel or two-channel configuration. In a one-channel configuration, the active electrode is placed at the high forehead ( $\mathrm{Cz}$ or Fpz), the reference electrode is placed at the ipsilateral earlobe or the mastoid, and the ground is placed at the contralateral earlobe or mastoid. In a two-channel configuration, the active electrodes for both channels are placed on the high forehead ( $\mathrm{Cz}$ or $\mathrm{Fpz}$ ), reference electrodes are placed on both earlobes or both mastoids, and the ground is placed at the center of the forehead.

o The number of samples averaged has ranged from 500 (Grinn et al., 2017) to 12,500 (Valderrama et al., 2018). Increasing the number of samples averaged will reduce noise in the ABR waveform, making it easier to resolve wave I, but data collection time is increased. It has been indicated that there is little improvement between 1000 and 2000 averages, except near threshold where as many as 4000 averages may be needed (Hall, 1992). It appears that 1000 averages is probably adequate when measuring responses to high level (90-100 dB SPL) click signals in a normal-hearing population, but increased averaging may be required when including participants with abnormal pure tone thresholds. A conservative approach would be to average a minimum of 4000 
responses; additional data would be helpful in guiding the minimum protocol requirements.

o Increasing the stimulus rate reduces neural recovery time between stimuli, reduces the ability to resolve wave I, and increases wave V latency. Stimulus presentation rates vary widely across studies. Hall (1992) shows that wave I amplitude is constant up to $21 / \mathrm{sec}$ rates and the amplitude decreases at $31 / \mathrm{sec}$ and at higher rates. Thus, a 21/sec rate would be recommended for a standard test rate; additional stimulus rates can be included to probe the rate of wave I amplitude decrease as stimulus rate increases.

o Although ABR measurements are a necessary element of the test battery, it must be noted that the field is not yet at a point where it is feasible to agree on whether wave I amplitude is the best metric or not, with some data suggesting that wave I is insensitive to low-SR fiber loss (Bourien et al., 2014).

Wave I has good test-retest reliability (low measurement error) but large between-subject variance (Mitchell et al., 1989; Don et al., 1994; Prendergast et al., 2018). A differential measure that reduces between-subject variance due to factors unrelated to synaptopathy is recommended for improved sensitivity. As discussed above, the use of a ratio derived from wave I (i.e., wave V/I, or SP/AP amplitude) or other metrics such as wave $\mathrm{V}$ latency may be problematic because (1) the value of the ratio critically depends on changes in the numerator and (2) wave $\mathrm{V}$ features reflect response characteristics from central auditory nuclei, which may or may not correlate with synaptopathy. The growth of ABR wave I with increasing stimulus intensity may be a useful differential wave I measure with reasonable test-retest reliability (Johannesen et al., 2019), but the data do not allow recommendation for a single best differential measure at this moment.

o In the absence of OHC deficits, it is not clear whether ABR latencies are impacted by synaptopathy. Delayed and temporally smeared first-spike latencies of low-SR fibers compared to highSR fibers mean that the ABR wave I is mostly dominated by the temporally precise high-SR fibers (Bourien et al., 2014). A selective loss of low-SR fibers or different degrees of high-SR fiber loss is thus not expected to impact the ABR wave-I latency much (see also simulations in Verhulst et al., 2018a). However, there is a suggestion that the degree to which the ABR wave-V latency shifts when background noise is added can be a marker of selective low/medium-SR fiber loss (Mehraei et al., 2016). In contrast to suggestions that ABR latencies reflect cochlear synaptopathy, it must also be noted that ABR latencies are very sensitive to $\mathrm{OHC}$ deficits and the shape of the audiogram (Gorga et al., 1985; Lewis et al., 2015). This means that ABR latencies for constant SPL stimulation can be used as a control measure to verify whether EHF loss contributed to the degraded ABR wave I amplitude. Specifically, the ABR waves would be delayed in listeners with OHC loss, when compared to listeners without OHC loss but with or without synaptopathy.

o It is important to control for any potential confounds due to high-frequency hair cell damage, which may impact wave I in particular (and more so than wave $V$ given that wave- $V$ generators are more low-frequency than the wave-I generators (Don and Eggermont, 1978)). EHF testing and/or high-frequency DPOAE measurements provide critical insight into peripheral damage and one or both of these measures should be included. Even in a sample with normal audiograms, it is advisable that ABR measures are statistically adjusted for between-subject differences in OAEs. OAEs are more sensitive to noise exposure than pure tone thresholds (Engdahl and Kemp, 1996; Seixas et al., 2005; Marshall et al., 2009) and OAEs measured in the high frequencies $(4-8 \mathrm{kHz})$ are correlated with pure tone thresholds in the extended high frequencies (11-20 kHz, Arnold et al., 1999). Given that OAEs are reflective of peripheral auditory function, adjusting ABR wave I amplitudes for OAEs may be preferable to adjusting for pure tone thresholds, which theoretically could be impacted by high levels of neuronal loss. Another potential method of limiting the impact of highfrequency hair cell damage is to add notched noise to the ABR stimulus. It should also be noted that wave I can be impacted by sub-clinical IHC dysfunction, and distinguishing synaptopathy from IHC dysfunction is problematic using wave I amplitude in isolation. It will also be problematic to distinguish synaptopathy (loss of synapses) from deafferentation (loss of nerve fibers) using wave I. In the absence of histopathology, which cannot be collected from live participants, we recommend that authors reporting results remain cognizant of these limitations and specifically acknowledge the imprecision of wave I results.

In summary, it is reasonable to infer there is a pathology of the IHCs, the synapses, or the ascending neural pathway, if middle ear conduction, $\mathrm{OHC}$ function, and threshold sensitivity (including EHF thresholds) are all normal, but there is decreased amplitude of the $\mathrm{AP}$ or wave I of the ABR. If middle ear conduction, OHC function, or threshold sensitivity are suspected to be compromised, the inference of selective synaptopathy is drawn into question. This does not mean synaptopathy has not occurred, but that functional deficits and/or suprathreshold complaints cannot be attributed to a selective neural pathology as there are other potentially contributing pathologies present.

There are a number of optional (experimental) elements of the test battery that labs may consider adding; it is possible that one or more of these elements will ultimately be identified as essential components to include in future investigations. These are described below.

- MEMR: also termed the acoustic reflex, stapedius reflex, or auditory reflex; this is an involuntary muscle contraction which can be triggered by either ipsilateral or contralateral sound. The AN must be intact to initiate the acoustic reflex; the strength of the acoustic reflex is reduced in mice with synaptopathy (Valero et al. 2016, 2018) and this may prove to be a useful metric in humans as well. MEMRs are known to be weak, or absent, in a subset of the population (Flamme et al., 2017; McGregor et al., 2018), and it has been suggested that synaptopathic injury could underlie this observed individual variability (Wojtczak et al., 2017). Use of a wideband probe and a broadband activator stimulus has been shown to lower MEMR thresholds compared to the standard $226 \mathrm{~Hz}$ probe tone used clinically, which could perhaps improve the ability to reliably detect MEMR responses in future studies (Feeney et al., 2017).

- Signal-in-Noise/Speech-in-Noise testing: Various labs are using different clinical tests, including the QuickSin, WIN, Matrix test, and others. The custom manipulation of the NU-6 words by Liberman et al. (2016) revealed significant differences in performance for high-risk and low-risk participants. However, this test is not readily available to others, complicating replication of the testing and reproduction of the results by other laboratories. Furthermore, task difficulty can be defined simply in terms of percent correct performance, which is easily manipulated in any speech-in-noise test by changing the signal-to-noise ratio. Making a test more "complex" with respect to the cues available would be expected to make the test more cognitively demanding (for example, requiring increased attention and listening effort). This is likely to make performance more reliant 
on central rather than peripheral factors, reducing sensitivity to synaptopathy.

- Testing audiometric thresholds for brief tones ( $<20 \mathrm{~ms}$ ) has been suggested as a possible method for detecting synaptopathy. Theoretical reasoning by Lopez-Poveda and Barrios (2013) and perceptual model simulations by Marmel et al. (2015) suggest that synaptopathy involves a substantial loss of lowthreshold AN fibers in addition to the larger loss of highthreshold fibers, which is predicted to elevate the detection threshold for brief tones, without significantly elevating the thresholds for longer sounds. The results of Wong et al. (2019) in the budgerigar undermine this approach and the experimental data currently available are not adequate to allow a recommended protocol for this test.

- Supra-threshold temporal tasks: Basic psychoacoustic tasks such as AM detection, temporal fine-structure sensitivity tasks, tone-in-noise detection, frequency and intensity discrimination as well as basic binaural ITD or ILD sensitivity tasks have been completed by some laboratories, but there is only limited theoretical development relating synaptopathy to specific deficits of interest. In those cases where deficits are present on only a subset of temporal processing tasks, interpretation is challenging. Some of these tests will be compromised by OHC pathology, highlighting the need for careful DPOAE assessment, if deficits are to be attributed to selective neural injury.

Hyperacusis tools: There are no uniform measures of hyperacusis; loudness discomfort levels could be considered for inclusion (following Bramhall et al., 2018), although this measure may not be a good predictor of hyperacusis. Alternatively, a measure of loudness growth, such as the Contour Test of Loudness Perception (Cox et al., 1997), or categorical loudness scaling (Brand and Hohmann, 2002), might be a better metric. Annoyance related to everyday sounds and avoidance of noisy environments (following Liberman et al., 2016) could be considered for inclusion as well.

- ABR amplitude versus latency plots can be derived from the raw data and may be considered as per Verhulst et al. (2016) to further disentangle the contribution of $\mathrm{OHC}$ and synaptopathy aspects to SNHL.

- EFR: The EFR is a steady-state sound evoked response which follows the envelope of an AM stimulus. The carrier and modulation frequency can be manipulated, as well as the depth of amplitude modulation. Some studies have also included masking noise (e.g., Bharadwaj et al., 2015; Paul et al., 2017). However, as described above, the EFR cannot be measured in humans easily at the high modulations rates $(\sim 1 \mathrm{kHz})$ that are associated with synaptopathy in animal models. Even though model simulations suggest that EFRs to lower modulation rates may also be sensitive to synaptopathy, the interpretation of the EFR metric in terms of synaptopathy might depend critically on the stimulus characteristics and masking noise applied.

Ultimately, to reach a definitive differential diagnosis of synaptopathy, we may need to turn to novel brain imaging techniques, perhaps variations of magnetic resonance imaging, positron emission tomography or magnetoencephalography, or a new method, such as the molecular imaging technique used to detect changes in the neurotransmitter dopamine in the human brain described by Badgaiyan (2014). It may be that research efforts into other neurological conditions, such as Alzheimer's disease, may yield viable techniques which hearing scientists can adopt for the detection of abnormal synaptic transmission at the AN.

The above list of suggestions for assays to detect synaptopathy is quite lengthy and would not be clinically feasible for diagnostic purposes due to time constraints. However, at this point in time it is not possible to minimize the number of assays because of the many uncertainties within the literature. A more concise battery of assays can only be suggested when the number of studies related to human synaptopathy increase and the combinations of assays become validated.

\section{Conclusions}

Despite a concerted international research effort over the past several years, conclusive evidence for noise-induced cochlear synaptopathy in humans remains elusive. In this commentary, we have discussed some of the possible reasons behind this. We have described how each of the various experimental approaches, including electrophysiological, questionnaire and behavioural measures have proved to be imperfect metrics. Although there may be techniques we can use to control variability, improve robustness, and increase statistical power, we seem far from reaching a satisfactory diagnostic approach. There are also important questions to be answered about the extent to which human synaptopathy mirrors the animal models, particularly in relation to the intensity of noise that is needed to induce synaptic damage in humans, the relative susceptibility of low-, medium- and high-SR fibers, and the possibility that structural repair at the synapse may occur following early auditory insults. Given that aging and cumulative noise exposure are necessarily correlated and associated with peripheral and central damage in addition to synaptopathy, disentangling noise-induced synaptopathy from deterioration of other auditory structures may prove to be an insurmountable challenge. Nevertheless, it is important to continue our efforts to determine whether synaptopathy occurs in humans, and to better understand its potential perceptual effects. As one of several peripheral and central factors that may contribute to suprathreshold hearing deficits in humans, we need to be able to characterize its relative influence on an individual's overall auditory function. Understanding these relationships is essential if we are to move beyond the audiogram towards a holistic model of person-specific hearing care that diagnoses and treats both the "hidden" and "unhidden" components that underlie human hearing impairment.

\section{Acknowledgements}

NB was supported by the Department of Veterans Affairs, Veterans Health Administration, Rehabilitation Research and Development Service - Award \#C2104-W (to NB). The opinions and assertions presented here are the private views of the authors and are not to be construed as official or as necessarily reflecting the views of the VA.

EFB was supported by the Australian Government Department of Health, the Hearing Industry Research Consortium, the National Health and Medical Research Council (Grant ID 1063905) and the HEARing CRC, established under the Australian Government's Cooperative Research Centres (CRC) Program. The CRC Program supports industry led collaborations between industry, researchers and the community.

EALP was supported by the Oticon Foundation, Junta de Castilla y León (grant SA023P17), Spanish Ministry of Economy and Competitiveness (grant BFU2015-65376-P) and the European Regional Development Fund.

CL is supported by the National Institute on Deafness and Other Communication Disorders (1R01DC014088), the Defense Medical Research and Development Program (W81XWH1820014), and the Emilie and Phil Schepps Professorship in Hearing Science. Opinions expressed here are those of the author.

$\mathrm{CP}$ was supported by the Medical Research Council (MR/ 
L003589/1 and MR/M023486/1), by the Biotechnology and Biological Sciences Research Council (BB/M007243/1), and by the NIHR Manchester Biomedical Research Centre.

BC is funded by Communication Disorders of the National Institutes of Health R21DC013172 and 1R56DC016415-01, the Swedish Medical Research Council K2014-99X-22478-01-3, Knut and Alice Wallenberg Foundation (B.C. \#KAW2008), Karolinska Institutet, Tysta Skolan, Hörselforskningsfonden, The Office of the Assistant Secretary of Defense for Health Affairs, through the Neurosensory and Rehabilitation under Award No. W81XWH-16-10032. Opinions, interpretations, conclusions and recommendations are those of the authors and are not necessarily endorsed by the Department of Defense.

SV was supported by the European Research Council under the Horizon 2020 Research and Innovation Programme (grant agreement No 678120 RobSpear).

\section{References}

Arnold, D.J., Lonsbury-Martin, B.L., Martin, G.K., 1999. High-frequency hearing influences lower-frequency distortion-product otoacoustic emissions. Arch. Otolaryngol. Head Neck Surg. 125, 215-222.

Badgaiyan, R.D., 2014. Imaging dopamine neurotransmission in live human brain. Prog. Brain Res. 211, 165-182.

Badri, R., Siegel, J.H., Wright, B.A., 2011. Auditory filter shapes and high-frequency hearing in adults who have impaired speech in noise performance despite clinically normal audiograms. J. Acoust. Soc. Am. 129, 852-863.

Bharadwaj, H.M., Masud, S., Mehraei, G., et al., 2015. Individual differences reveal correlates of hidden hearing deficits. J. Neurosci. 35, 2161-2172.

Bharadwaj, H.M., Verhulst, S., Shaheen, L., et al., 2014. Cochlear neuropathy and the coding of supra-threshold sound. Front. Syst. Neurosci. 8, 26.

Bourien, J., Tang, Y., Batrel, C., et al., 2014. Contribution of auditory nerve fibers to compound action potential of the auditory nerve. J. Neurophysiol. 112, 1025-1039.

Bramhall, N., Ong, B., Ko, J., et al., 2015. Speech perception ability in noise is correlated with auditory brainstem response wave I amplitude. J. Am. Acad. Audiol. 26, 509-517.

Bramhall, N.F., Konrad-Martin, D., McMillan, G.P., 2018. Tinnitus and auditory perception after a history of noise exposure: relationship to auditory brainstem response measures. Ear Hear. 39, 881-894.

Bramhall, N.F., Konrad-Martin, D., McMillan, G.P., et al., 2017. Auditory brainstem response altered in humans with noise exposure despite normal outer hair cell function. Ear Hear. 38, e1-e12.

Brand, T., Hohmann, V., 2002. An adaptive procedure for categorical loudness scaling. J. Acoust. Soc. Am. 112, 1597-1604.

Carney, L.H., 2018. Supra-threshold hearing and fluctuation profiles: implications for sensorineural and hidden hearing loss. J. Assoc. Res. Otolaryngol. 19, $331-352$.

Chen, G.D., Fechter, L.D., 2003. The relationship between noise-induced hearing loss and hair cell loss in rats. Hear. Res. 177, 81-90.

Colquhoun, D., 2017. The reproducibility of research and the misinterpretation of pvalues. Royal Society open science 4, 171085.

Cox, R.M., Alexander, G.C., Taylor, I.M., et al., 1997. The contour test of loudness perception. Ear Hear. 18, 388-400.

Dallos, P., Zheng, J., Cheatham, M.A., 2006. Prestin and the cochlear amplifier J. Physiol. 576, 37-42.

Dobie, R.A., Humes, L.E., 2017. Commentary on the regulatory implications of noiseinduced cochlear neuropathy. Int. J. Audiol. 56, 74-78.

Dolphin, W.F., Mountain, D.C., 1992. The envelope following response: scalp potentials elicited in the Mongolian gerbil using sinusoidally AM acoustic signals. Hear. Res. 58, 70-78.

Don, M., Eggermont, J.J., 1978. Analysis of the click-evoked brainstem potentials in man unsing high-pass noise masking. J. Acoust. Soc. Am. 63, 1084-1092.

Don, M., Ponton, C.W., Eggermont, J.J., et al., 1994. Auditory brainstem response (ABR) peak amplitude variability reflects individual differences in cochlear response times. J. Acoust. Soc. Am. 96, 3476-3491.

Encina-Llamas, G., Parthasarathy, A., Harte, J., et al., 2017. Synaptopathy with envelope following responses (EFRs): the off-frequency problem. In: International Symposium on Auditory and Audiological Research (ISAAR). Nyborg, Denmark.

Engdahl, B., Kemp, D.T., 1996. The effect of noise exposure on the details of distortion product otoacoustic emissions in humans. J. Acoust. Soc. Am. 99, 1573-1587.

Feeney, M.P., Keefe, D.H., Hunter, L.L., et al., 2017. Normative wideband reflectance, equivalent admittance at the tympanic membrane, and acoustic stapedius reflex threshold in adults. Ear Hear. 38, e142-e160.

Felder, E., Schrott-Fischer, A., 1995. Quantitative evaluation of myelinated nerve fibres and hair cells in cochleae of humans with age-related high-tone hearing loss. Hear. Res. 91, 19-32.

Fernandez, K.A., Jeffers, P.W., Lall, K., et al., 2015. Aging after noise exposure: acceleration of cochlear synaptopathy in "recovered" ears. J. Neurosci. 35, 7509-7520.

Flamme, G.A., Deiters, K.K., Tasko, S.M., et al., 2017. Acoustic reflexes are common but not pervasive: evidence from the National Health and Nutrition Examination Survey, 1999-2012. Int. J. Audiol. 56, 52-62.

Fulbright, A.N.C., Le Prell, C.G., Griffiths, S.K., et al., 2017. Effects of recreational noise on threshold and suprathreshold measures of auditory function. Semin. Hear. 38, 298-318.

Fullgrabe, C., Moore, B.C., Stone, M.A., 2014. Age-group differences in speech identification despite matched audiometrically normal hearing: contributions from auditory temporal processing and cognition. Front. Aging Neurosci. 6, 347.

Furman, A.C., Kujawa, S.G., Liberman, M.C., 2013. Noise-induced cochlear neuropathy is selective for fibers with low spontaneous rates. J. Neurophysiol. 110, 577-586.

Gilles, A., Schlee, W., Rabau, S., et al., 2016. Decreased speech-in-noise understanding in young adults with tinnitus. Front. Neurosci. 10, 288.

Gorga, M.P., Neely, S.T., Ohlrich, B., et al., 1997. From laboratory to clinic: a large scale study of distortion product otoacoustic emissions in ears with normal hearing and ears with hearing loss. Ear Hear. 18, 440-455.

Gorga, M.P., Worthington, D.W., Reiland, J.K., et al., 1985. Some comparisons between auditory brain stem response thresholds, latencies, and the pure-tone audiogram. Ear Hear. 6, 105-112.

Grinn, S.K., Wiseman, K.B., Baker, J.A., et al., 2017. Hidden hearing loss? No effect of common recreational noise exposure on cochlear nerve response amplitude in humans. Front. Neurosci. 11, 465.

Grose, J.H., Buss, E., Hall 3rd, J.W., 2017. Loud music exposure and cochlear synaptopathy in young adults: isolated auditory brainstem response effects but No perceptual consequences. Trends. Hear. 21, 2331216517737417.

Gu, J.W., Herrmann, B.S., Levine, R.A., et al., 2012. Brainstem auditory evoked potentials suggest a role for the ventral cochlear nucleus in tinnitus. J. Assoc. Res. Otolaryngol 13, 819-833.

Guest, H., Munro, K.J., Plack, C.J., 2017a. Tinnitus with a normal audiogram: role of high-frequency sensitivity and reanalysis of brainstem-response measures to avoid audiometric over-matching. Hear. Res. 356, 116-117.

Guest, H., Munro, K.J., Prendergast, G., et al., 2017b. Tinnitus with a normal audiogram: relation to noise exposure but no evidence for cochlear synaptopathy. Hear. Res. 344, 265-274.

Guest, H., Munro, K.J., Prendergast, G., et al., 2018. Impaired speech perception in noise with a normal audiogram: No evidence for cochlear synaptopathy and no relation to lifetime noise exposure. Hear. Res. 364, 142-151.

Hall, J.W.I., 1992. Handbook of Auditory Evoked Responses. Allyn and Bacon, Boston.

Henry, J.A., Roberts, L.E., Caspary, D.M., et al., 2014. Underlying mechanisms of tinnitus: review and clinical implications. J. Am. Acad. Audiol. 25, 5-22 quiz 126.

Hickox, A.E., Larsen, E., Heinz, M.G., et al., 2017. Translational issues in cochlear synaptopathy. Hear. Res. 349, 164-171.

Holtegaard, P., Epp, B., 2018. Correlation of ABR wave I level-growth and amplitude modulation discrimination in listeners with poor word recognition in noise. In: ARO MidWinter Meeting. San Diego, CA.

Hopkins, K., Moore, B.C., 2011. The effects of age and cochlear hearing loss on temporal fine structure sensitivity, frequency selectivity, and speech reception in noise. J. Acoust. Soc. Am. 130, 334-349.

Johannesen, P.T., Buzo, B.C., Lopez-Poveda, E.A., 2019. Evidence for age-related cochlear synaptopathy in humans unconnected to speech-in-noise intelligibility deficits. Hear. Res. 374, 35-48.

Johannesen, P.T., Perez-Gonzalez, P., Kalluri, S., et al., 2016. The influence of cochlear mechanical dysfunction, temporal processing deficits, and age on the intelligibility of audible speech in noise for hearing-impaired listeners. Trends Hear 20.

Kimura, R.S., Ota, C.Y., Takahashi, T., 1979. Nerve fiber synapses on spiral ganglion cells in the human cochlea. Ann. Otol. Rhinol. Laryngol. 88, 1-17.

Kujawa, S.G., Liberman, M.C., 2009. Adding insult to injury: cochlear nerve degeneration after "temporary" noise-induced hearing loss. J. Neurosci. 29, $14077-14085$.

Kujawa, S.G., Liberman, M.C., 2015. Synaptopathy in the noise-exposed and aging cochlea: primary neural degeneration in acquired sensorineural hearing loss. Hear. Res. 330, 191-199.

Le Prell, C.G., 2019. Effects of noise exposure on auditory brainstem response and speech-in-noise tasks: a review of the literature. Int. J. Audiol. 58, S3-S32.

Le Prell, C.G., Siburt, H.W., Lobarinas, E., et al., 2018. no reliable association between recreational noise exposure and threshold sensitivity, distortion product otoacoustic emission amplitude, or word-in-noise performance in a college student Population. Ear Hear 39, 1057-1074.

Lewis, J.D., Kopun, J., Neely, S.T., et al., 2015. Tone-burst auditory brainstem response wave V latencies in normal-hearing and hearing-impaired ears. J. Acoust. Soc Am. 138, 3210-3219.

Liberman, M.C., Epstein, M.J., Cleveland, S.S., et al., 2016. Toward a differentia diagnosis of hidden hearing loss in humans. PLoS One 11 e0162726.

Lin, H.W., Furman, A.C., Kujawa, S.G., et al., 2011. Primary neural degeneration in the Guinea pig cochlea after reversible noise-induced threshold shift. J Assoc Res Otolaryngol 12, 605-616.

Liu, C., Bu, X., Wu, F., et al., 2012. Unilateral auditory neuropathy caused by cochlear nerve deficiency. Int I Otolaryngol 914986, 2012.

Lobarinas, E., Salvi, R., Ding, D., 2016. Selective inner hair cell dysfunction in chinchillas impairs hearing-in-noise in the absence of outer hair cell loss. J Assoc Res 
Otolaryngol 17, 89-101.

Lobarinas, E. Spankovich, C. Le Prell, C.G. 2017. Evidence of "hidden hearing loss" following noise exposures that produce robust TTS and ABR wave-I amplitude reductions. Hear. Res. 349, 155-163.

Lopez-Poveda, E.A., 2014. Why do I hear but not understand? Stochastic undersampling as a model of degraded neural encoding of speech. Front. Neurosci. 8 348.

Lopez-Poveda, E.A., Barrios, P., 2013. Perception of stochastically undersampled sound waveforms: a model of auditory deafferentation. Front. Neurosci. 7,124.

Lopez-Poveda, E.A., Johannesen, P.T. Perez-Gonzalez, P. et al., 2017. Predictors of hearing-aid outcomes. Trends Hear 21, 2331216517730526.

Makary, C.A., Shin, J., Kujawa, S.G., et al., 2011. Age-related primary cochlear neuronal degeneration in human temporal bones. J Assoc Res Otolaryngol 12 $711-717$.

Marmel, F., Rodríguez-Mendoza, M.A., Lopez-Poveda, E.A., 2015. Stochastic undersampling steepens auditory threshold/duration functions: implications for understanding auditory deafferentation and aging. Front. Aging Neurosci. 7, 63.

Marshall, L., Lapsley Miller, J.A., Heller, L.M., et al., 2009. Detecting incipient innerear damage from impulse noise with otoacoustic emissions. J. Acoust. Soc. Am. 125, 995-1013.

McGregor, K.D., Flamme, G.A., Tasko, S.M., et al., 2018. Acoustic reflexes are common but not pervasive: evidence using a diagnostic middle ear analyser. Int. J. Audiol 57. S42-S50.

Mehraei, G., Hickox, A.E., Bharadwaj, H.M., et al., 2016. Auditory brainstem response latency in noise as a marker of cochlear synaptopathy. J. Neurosci. 36 $3755-3764$.

Melcher, J.R., Kiang, N.Y., 1996. Generators of the brainstem auditory evoked potential in cat. III: identified cell populations. Hear. Res. 93, 52-71.

Mitchell, C., Phillips, D.S., Trune, D.R., 1989. Variables affecting the auditory brainstem response: audiogram, age, gender and head size. Hear. Res, 40, 75-85.

Möhrle, D., Ni, K., Varakina, K., et al., 2016. Loss of auditory sensitivity from inne hair cell synaptopathy can be centrally compensated in the young but not old brain. Neurobiol. Aging 44,173-184.

Nadol Jr, J.B., 1988. Comparative anatomy of the cochlea and auditory nerve in mammals. Hear. Res. 34, 253-266.

Otte, J., Schunknecht, H.F., Kerr, A.G., 1978. Ganglion cell populations in normal and pathological human cochleae. Implications for cochlear implantation. Laryngoscope 88, 1231-1246.

Oxenham, A.J., 2016. Predicting the perceptual consequences of hidden hearing loss Trends Hear 20, 2331216516686768.

Parthasarathy, A., Kujawa, S.G., 2018. Synaptopathy in the aging cochlea: characterizing early-neural deficits in auditory temporal envelope processing. J. Neurosci. 38, 7108-7119.

Paul, B.T., Bruce, I.C., Roberts, L.E., 2017. Evidence that hidden hearing loss underlies amplitude modulation encoding deficits in individuals with and without tinnitus. Hear. Res. 344, 170-182.

Prendergast, G., Guest, H., Munro, K.J., et al., 2017a. Effects of noise exposure on young adults with normal audiograms I: Electrophysiology. Hear. Res. 344 68-81.

Prendergast, G., Millman, R.E., Guest, H., et al., 2017b. Effects of noise exposure on young adults with normal audiograms II: behavioral measures. Hear. Res. 356 $74-86$.

Prendergast, G., Tu, W., Guest, H., et al., 2018. Supra-threshold auditory brainstem response amplitudes in humans: test-retest reliability, electrode montage and noise exposure. Hear. Res, 364, 38-47.

Rasetshwane, D.M., Argenyi, M., Neely, S.T., et al., 2013. Latency of tone-burstevoked auditory brain stem responses and otoacoustic emissions: level, frequency, and rise-time effects. J. Acoust. Soc. Am. 133, 2803-2817.

Ridley, C.L., Kopun, J.G., Neely, S.T., et al., 2018. Using thresholds in noise to identify hidden hearing loss in humans. Ear Hear. 39, 829-844.

Roberts, L.E., Paul, B.T., Bruce, I.C., 2018. Erratum and comment: envelope following responses in normal hearing and in tinnitus. Hear. Res. 361, 157-158.

Salvi, R., Sun, W., Ding, D., et al., 2017. Inner hair cell loss disrupts hearing and cochlear function leading to sensory deprivation and enhanced central auditory gain. Front. Neurosci. 10, 621.

Salvi, R., Sun, W., Ding, D., et al., 2016. Inner hair cell loss disrupts hearing and cochlear function leading to sensory deprivation and enhanced central auditory gain. Front. Neurosci. 10, 621

Sato, H., Sando, I., Takahashi, H., 1991. Sexual dimorphism and development of the human cochlea. Computer 3-D measurement. Acta Otolaryngol. 111, 1037-1040.

Schaette, R., McAlpine, D., 2011. Tinnitus with a normal audiogram: physiological evidence for hidden hearing loss and computational model. J. Neurosci. 31, $13452-13457$.

Seixas, N., Goldman, B., Sheppard, L., et al., 2005. Prospective noise induced changes to hearing among construction industry apprentices. Occup. Environ. Med. 62, $309-317$.

Sergeyenko, Y., Lall, K., Liberman, M.C., et al., 2013. Age-related cochlear synaptopathy: an early-onset contributor to auditory functional decline. J. Neurosci. 33, 13686-13694.

Shaheen, L.A., Valero, M.D., Liberman, M.C., 2015. Towards a diagnosis of cochlear neuropathy with envelope following responses. J Assoc Res Otolaryngol 16, $727-745$.

Sheldrake, J., Diehl, P.U., Schaette, R., 2015. Audiometric characteristics of hyperacusis patients. Front. Neurol. 6, 105.

Shi, L., Chang, Y., Li, X., et al., 2016. Coding deficits in noise-induced hidden hearing loss may stem from incomplete repair of ribbon synapses in the cochlea. Front. Neurosci. 10, 231.

Shim, H.J., An, Y.H., Kim, D.H., et al., 2017. Comparisons of auditory brainstem response and sound level tolerance in tinnitus ears and non-tinnitus ears in unilateral tinnitus patients with normal audiograms. PLoS One 12 e0189157.

Singer, W., Kasini, K., Manthey, M., et al., 2018. The glucocorticoid antagonist mifepristone attenuates sound-induced long-term deficits in auditory nerve response and central auditory processing in female rats. FASEB J. 32, 3005-3019.

Skoe, E., Tufts, J., 2018. Evidence of noise-induced subclinical hearing loss using auditory brainstem responses and objective measures of noise exposure in humans. Hear. Res. 361, 80-91.

Spankovich, C., Le Prell, C.G., Lobarinas, E., et al., 2017. Noise history and auditory function in young adults with and without type 1 diabetes mellitus. Ear Hear. 38, 724-735.

Stamper, G.C., Johnson, T.A., 2015a. Auditory function in normal-hearing, noiseexposed human ears. Ear Hear. 36, 172-184.

Stamper, G.C., Johnson, T.A., 2015b. Letter to the editor: examination of potential sex influences in Stamper, G. C., \& Johnson, T.A. (2015). Auditory function in normal-hearing, noise-exposed human ears, ear hear, 36, 172-184. Ear Hear. 36, $738-740$.

Stone, M.A., Moore, B.C., 2014. Amplitude-modulation detection by recreationalnoise-exposed humans with near-normal hearing thresholds and its mediumterm progression. Hear. Res. 317, 50-62.

Strelcyk, O., Dau, T., 2009. Relations between frequency selectivity, temporal finestructure processing, and speech reception in impaired hearing. J. Acoust. Soc. Am. 125, 3328-3345.

Subramaniam, M., Henderson, D., Spongr, V., 1994a. The relationship among distortion-product otoacoustic emissions, evoked potential thresholds, and outer hair cells following interrupted noise exposures. Ear Hear. 15, 299-309.

Subramaniam, M., Salvi, R.J., Spongr, V.P., et al., 1994b. Changes in distortion product otoacoustic emissions and outer hair cells following interrupted noise exposures. Hear. Res. 74, 204-216.

Thong, J.F., Low, D., Tham, A., et al., 2017. Cochlear duct length-one size fits all? Am. J. Otolaryngol. 38, 218-221.

Valderrama, J.T. Beach, E.F., Yeend, I., et al., 2018. Effects of lifetime noise exposure on the middle-age human auditory brainstem response, tinnitus and speech-innoise intelligibility. Hear. Res. 365, 36-48.

Valero, M.D., Burton, J.A., Hauser, S.N., et al., 2017. Noise-induced cochlear synaptopathy in rhesus monkeys (Macaca mulatta). Hear. Res. 353, 213-223.

Valero, M.D., Hancock, K.E., Liberman, M.C., 2016. The middle ear muscle reflex in the diagnosis of cochlear neuropathy. Hear. Res. 332, 29-38.

Valero, M.D., Hancock, K.E., Maison, S.F., et al., 2018. Effects of cochlear synaptopathy on middle-ear muscle reflexes in unanesthetized mice. Hear. Res. 363, 109-118.

Verhulst, S., Altoe, A., Vasilkov, V., 2018a. Computational modeling of the human auditory periphery: auditory-nerve responses, evoked potentials and hearing loss. Hear. Res. 360, 55-75.

Verhulst, S., Ernst, F., Garrett, M., et al., 2018b. Supra-threshold psychoacoustics and envelope-following response relations: normal-hearing, synaptopathy and cochlear gain loss. Acta Acustica united Acustica 104, 800-804.

Verhulst, S., Jagadeesh, A., Mauermann, M., et al., 2016. Individual differences in auditory brainstem response wave characteristics: relations to different aspects of peripheral hearing loss. Trends Hear 20.

Viana, L.M., O'Malley, J.T., Burgess, B.J., et al., 2015. Cochlear neuropathy in human presbycusis: confocal analysis of hidden hearing loss in post-mortem tissue. Hear. Res. 327, 78-88.

Wojtczak, M., Beim, J.A., Oxenham, A.J., 2017. Weak middle-ear-muscle reflex in humans with noise-induced tinnitus and normal hearing may reflect cochlear synaptopathy. eNeuro 4.

Wong, S.J., Abrams, K.S., Amburgey, K.N., et al., 2019. Effects of selective auditorynerve damage on the behavioral audiogram and temporal integration in the budgerigar. Hear. Res. 374, 24-34.

Wu, P.Z., Liberman, L.D., Bennett, K., et al., 2018. Primary neural degeneration in the human cochlea: evidence for hidden hearing loss in the aging ear. Neuroscience (in press). https://doi.org/10.1016/j.neuroscience.2018.07.053.

Yeend, I., Beach, E.F., Sharma, M., et al., 2017. The effects of noise exposure and musical training on suprathreshold auditory processing and speech perception in noise. Hear. Res. 353, 224-236.

Zaugg, T.L., Thielman, E.J., Griest, S., et al., 2016. Subjective reports of trouble tolerating sound in daily life versus loudness discomfort levels. Am. J. Audiol. $25,359-363$.

Zilany, M.S., Bruce, I.C., Carney, L.H., 2014. Updated parameters and expanded simulation options for a model of the auditory periphery. J. Acoust. Soc. Am. $135,283-286$. 